INTERNATIONAL PHASE OF OCEAN DRILLING (IPOD)

DEEP SEA DRILLING PROJECT

DEVELOPMENT ENGINEERING

TECHNICAL NOTE NO. 5

\title{
CORE BARREL INSTRUMENTATION, PRESSURE (CBIP)
}




\section{DISCLAIMER}

This report was prepared by the Deep Sea Drilling Project, Eniversity of Cal ifornia, San Diego, as an account of work sponsored by the United States Government's National Science Foundation, Neither the University nor any of their employees, nor any of their contractors, subcontractors, or their employees, makes any warranty, express or implfed, or assumes any legal liability or responsibility for the accuracy, completeness or usefulness of any information, apparatus, product or process disclosed, or represents that Its use would not infringe privately owned rights. 


\title{
TECHNICAL NOTE NO. 5
}

CORE BARREL INSTRUMENTATION, PRESSURE (CBIP)

\author{
Prepared for the \\ NATIONAL SCIENCE FOUNDATION \\ National Ocean Sediment Coring Program \\ Under Contract C-482 \\ by the \\ UNIVERSITY OF CALIFORNIA \\ Scripps Institution of Oceanography \\ Prime Contractor for the Project
}

June 1984

W. A. Nierenberg, Director

Scripps Institution of Oceanography
M. N. A. Peterson

Principal Investigator and

Project Manager

Deep Sea Drilling Project

Scripps Institution of Oceanography 


\section{$\underline{\text { INTRODUCTION }}$}

\section{CORE BARREL INSTRUMENTATION, PRESSURE (CBIP)}

DSDP has been using core barrels of various types for 15 years to retrieve samples of the deep ocean bottom. However, the hydraulic environment in and around the core barrel is still not understood too well. The CBIP was designed and built to measure pressures at some chosen locations in the core barrel during the coring process. Once the pressure regime has been determined it may be possible to develop more efficient coring systems.

The CBIP is presently set up to make three separate pressure measurements-at the top of the core barrel, above the ball vent valve, and in the annulus between the core barrel and the drill pipe. The tool is rated to 10,000 psi $(22,500$ feet $)$. The electronics contains three channels and is expandable to six channels. The data sampling rate can be set from one sample every 5.12 seconds to one sample every .32 second. At the slowest sampling rate the memory unit will provide for approximately 8 hours of recording time using the three channels. 
CORE BARREL INSTRUMENTATION, PRESSURE (CBIP)

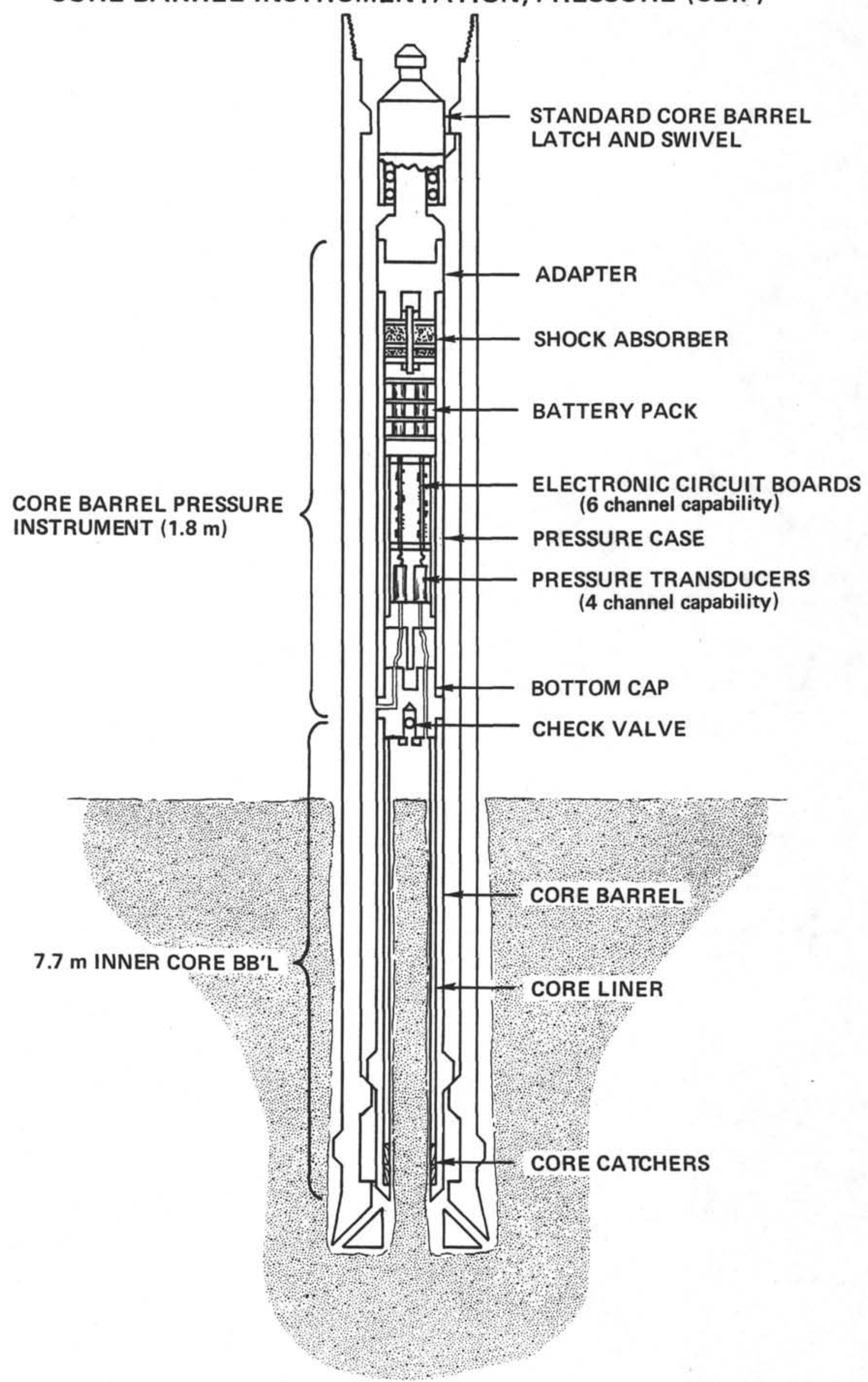




\section{INDEX}

I. DEPLOYMENT OF CORE BARREL INSTRUMENTATION, PRESSURE (CBIP)

II. INSTRUCTIONS FOR ASSEMBLY OF CBIP

III. PARTS LIST

IV. DRAWINGS

V. APPENDICES

A. PRESSURE MEASUREMENT TOOL RECORDER PACKAGE SPECIFICATIONS

B. TRANSDUCER SPECIFICATIONS

C. TRANSDUCER CALIBRATIONS

D. MEASURED DATA 
The CBIP has been deployed on two legs of the DSDP--Leg 94 and Leg 96 . On Leg 96 , the calibration was improper, and no meaningful data was obtained from the one run. On Leg 94, the CBIP was run four times, all with the Extended Core Barrel (XCB) system. The data from the first two runs, when plotted, showed lags in the response of the transducers caused by grease blockages in the small pressure tubing leading from the ports to the transducers. This data, also, was considered not meaningful. The grease was cleared out of the tubing and provision was made to prevent any more grease from entering the tubing.

The last two runs of the CBIP, after the grease blockages were removed, yielded very good data. The plots of this data, labeled No. 3 and No. 4, are included in this package. Plot No. 3 shows the increasing pressure as the instrument descended to the bottom of the drill string. After landing, but before coring, P2=P3 and both were greater than P1 by about 270 psi. When coring started, the ball vent valve lifted against the 270 psi back pressure and $\mathrm{P} 1=\mathrm{P} 2=\mathrm{P} 3$ at about 4600 psi. After coring was complete, the ball valve closed again and $\mathrm{P} 2=\mathrm{P} 3>\mathrm{P} 1$. When coring and circulation were stopped to make a connection, the pressures equalized at ambient pressure (about 4350 psi). A similar situation occurred each time a connection was made. Finally, the plot shows decreasing pressure as the instrument was hauled back up to the surface.

Plot No. 4 shows events similar to those of Plot No. 3. However, one event occurred in run No. 4 that did not occur in run No. 3 . That is the pressure spike just before the instrument was returned to the surface. It is believed that this spike was due to the impact of the overshot landing on top of the core barrel.

The data from these deployments of the CBIP have shown:

1) The drop rate of the core barrel is around 10 to 15 feet per second.

2) Because of the circulation a back pressure of 250 to 300 psi is imposed upon the ball vent before coring is started. This pressure must be overcome, and the ball lifted, before water can vent off and core can be taken into the core barrel. Actually, at the start of coring, the water may be able to vent down around the outside of the core, but as the core becomes longer, this vent path becomes more restrictive.

3) The pressures $P 2$ and $P 3$ are essentially equal throughout the entire operation except for the trip back to the surface where P2 exceeds P3 by about 100 psi. The reason for this is not clear. The two pressure regions P2 and P3 are interconnected and should be equal. As the core barrel is being retrieved, the downward relative flow of water in the annulus may tend to produce a venturi effect in the region of $\mathrm{P} 3$, but it seems that this would produce, if anything, a lower pressure for P2. The dynamic pressure that would result from the velocity of retrieval is about 0.5 psi--nowhere near the 100 psi difference between P2 and P3. 
The following recommendations are put forth for future work involving the CBIP:

1) The instrument has only been run with the XCB system. The attempt to run it with the standard rotary coring system on Leg 94 did not produce meaningful data. More attempts should be made with the rotary system.

2) It would be useful to make a few runs of the CBIP without the ball in the venting system. It needs to be determined if the ball valve is really necessary, and if so, during what part of the operation it is needed. Perhaps some other arrangement could be set up whereby the top of the core barrel is freely vented (with no back pressure) during coring and then is closed of during retrieval to prevent washing of the core.

Don Rullivis

Don Bellows 


\section{INSTRUCTIONS FOR ASSEMBLY OF \\ CORE BARREL INSTRUMENTATION, PRESSURE (CBIP)}

Refer to Assemb1y Drawing R-OP3330. The CBIP can be used with the Standard Rotary Coring System or with the Extended Core Barrel (XCB).

1. Install 0-Ring, Backup Ring and Rubber Shock Pad (OP3350) in Bottom Cap (OP3349). Install 0-Ring and Backup Ring in Adapter (OP3331).

2. Install desired number of Pressure Transducers (1 to 4 ) on Transducer Block (OP3345). Plug unused ports on Transducer Block with Swagelok Plugs. CBIP is designed to allow any one or all of three pressure measurements at top of core barrel, above ball valve, and in annulus between core barrel and drill pipe.

3. Assemble Bottom Cap to Torque Rod (OP3346) by means of Torque Rod Plate (OP3347) and Torque Rod Cap (OP3348).

4. Install 1/16 0.D. Pressure Tubing (OP3353) as required. P1ug unused ports on top face of Bottom Cap with Swagelok Plugs. Plug unused pipe threaded ports with pipe plugs. From this point on, be careful that Pressure Tubing is not damaged by subsequent handling.

5. Attach half of Holding Tray (OP3342) to Transducer Block at one end and to Hang Plate (OP3338) at other end. Transducer Block goes at end nearest small holes (for mounting Electronics Package). Install Shock Rod (OP3333).

6. Install Electronics Pack and Battery Pack into Holding Tray and attach at bulkheads with screws as shown. Shock Indicator is optional--it may or may not be installed. Make appropriate electrical connections between Battery Pack, Electronics Pack, and Pressure Transducers. Set timer (if tool is to be deployed soon) and install other half of Holding Tray.

7. Slide Rebound Pad (OP3337) on to Shock Rod. Apply coating of silicone grease to I.D. of Pressure Case (OP3340) where Bottom Cap 0-rings will contact. Also, apply Moly-D grease to threads on Bottom Cap and Adapter.

8. Install inner assembly into Pressure Case by sliding in from end opposite previously installed Stop Plate (OP3336). Shock Rod will pass thru hole in center of Stop Plate and appear near end of Pressure Case. Screw Bottom Cap into Pressure Case and tighten securely with strap wrenches. If Shock Indicator has been installed, be careful when handling and wrenching that large shocks are not applied which might cause the Shock Indicator to trip prematurely. 
9. Pull end of Shock Rod until instrument package is fully extended on Torque Rod. Install Shock Sleeve (OP-3352), Foam Shock Pad (OP-3335), and Moving P1ate (OP-3334). Then install locknut on Shock Rod.

10. Apply coating of silicone grease to I.D. of Pressure Case where Adapter 0-Rings will contact. Install Adapter and wrench down securely with strap wrenches. Shock Indicator is optional.

11. To use CBIP in Rotary Core Barrel, remove Thread Protector (OP-3351). Bottom end of CBIP attaches into top of core barrel; top end attaches into swivel. Length of CBIP, shoulder to shoulder, is six feet. To use in Extended Core Barrel (XCB) a double box sub (OP-3236) must be used at bottom of CBIP to mate with Vent Sub. A double pin sub (OP-4401) must be used at top of CBIP to mate with Quick Release Assembly.

There is no need for roughnecks to apply wrench to Pressure Case $(\mathrm{OP}-3340)$ and it should not be done. Wrench should be applied only to Adapter $(\mathrm{OP}-3331)$ or Bottom Cap (OP-3349) when connecting CBIP to core barrel.

The CBIP will be shipped completely assembled. To remove instrument package from pressure housing, first take off Adapter. This will expose locknut on end of Shock Rod. Remove locknut. Entire instrument package may then be removed by unscrewing Bottom Cap and pulling from bottom end. 
PARTS LIST - CORE BARREL INSTRUMENTATION, PRESSURE (OP3330)

\begin{tabular}{|c|c|c|c|c|}
\hline ITEM NO. & DESCRIPTION & PART NO. & REQ' D & SUPPLIER \\
\hline 1 & Adapter & OP-3331 & 1 & \\
\hline 2 & Mount, Shock Indicator (Optiona1) & OP-3332 & 2 & \\
\hline 3 & Shock Rod & $\mathrm{OP}-3333$ & 1 & \\
\hline 4 & Moving Plate & OP-3334 & 1 & \\
\hline 5 & Shock Pad, Foam & $\mathrm{OP}-3335$ & 1 & \\
\hline 6 & Stop Plate & OP-3336 & 1 & \\
\hline 7 & Rebound Pad & OP-3337 & 1 & \\
\hline 8 & Hang Plate & OP-3338 & 1 & \\
\hline 9 & Plate, Battery & OP-3339 & 2 & \\
\hline 10 & Pressure Case & OP-3340 & 1 & \\
\hline 11 & Plate, Insulator & OP-3341 & 4 & \\
\hline 12 & Holding Tray & $\mathrm{OP}-3342$ & 1 & \\
\hline 14 & Electronics Plate & OP-3344 & 2 & \\
\hline 15 & Transducer Block & OP-3345 & 1 & \\
\hline 16 & Torque Rod & $\mathrm{OP}-3346$ & 1 & \\
\hline 17 & Plate, Torque Rod & OP-3347 & 1 & \\
\hline 18 & Cap, Torque Rod & $0 P-3348$ & 1 & \\
\hline 19 & Bottom Cap & OP-3349 & 1 & \\
\hline 20 & Shock Pad, Rubber & $\mathrm{OP}-3350$ & 1 & \\
\hline 21 & Thread Projector & OP-3351 & 1 & \\
\hline 22 & Shock Sleeve (optional) & OP-3352 & 1 & \\
\hline 23 & Pressure Tubing & OP-3353 & $1-4$ & \\
\hline 24 & P.C. Board Assembly & OP-3354 & 1 & \\
\hline 25 & ADC-RAM Board & $0 P-3355$ & 1 & Tex Engrg (S.D.) \\
\hline 26 & Logic Board & OP-3356 & 1 & Tex Engrg (S.D.) \\
\hline 27 & Torque Rod Assembly & $\mathrm{OP}-3357$ & 1 & \\
\hline 28 & Tie Rod & OP-3358 & 2 & \\
\hline 29 & Valve Seat Retainer & OP-3359 & 1 & \\
\hline 30 & Pressure Transducer & CEC -1200 & $1-4$ & Be11 \& Howe11, CEC Div (L.A.) \\
\hline 31 & Connector & CEC $166267-0006$ & $1-4$ & Bel1 \& Howe11, CEC Div (L.A.) \\
\hline 32 & Battery, Gates 2 Volt, 2.5 AH D Cell & 0810-0004 & 8 & Tauber Electronics (S.D.) \\
\hline
\end{tabular}




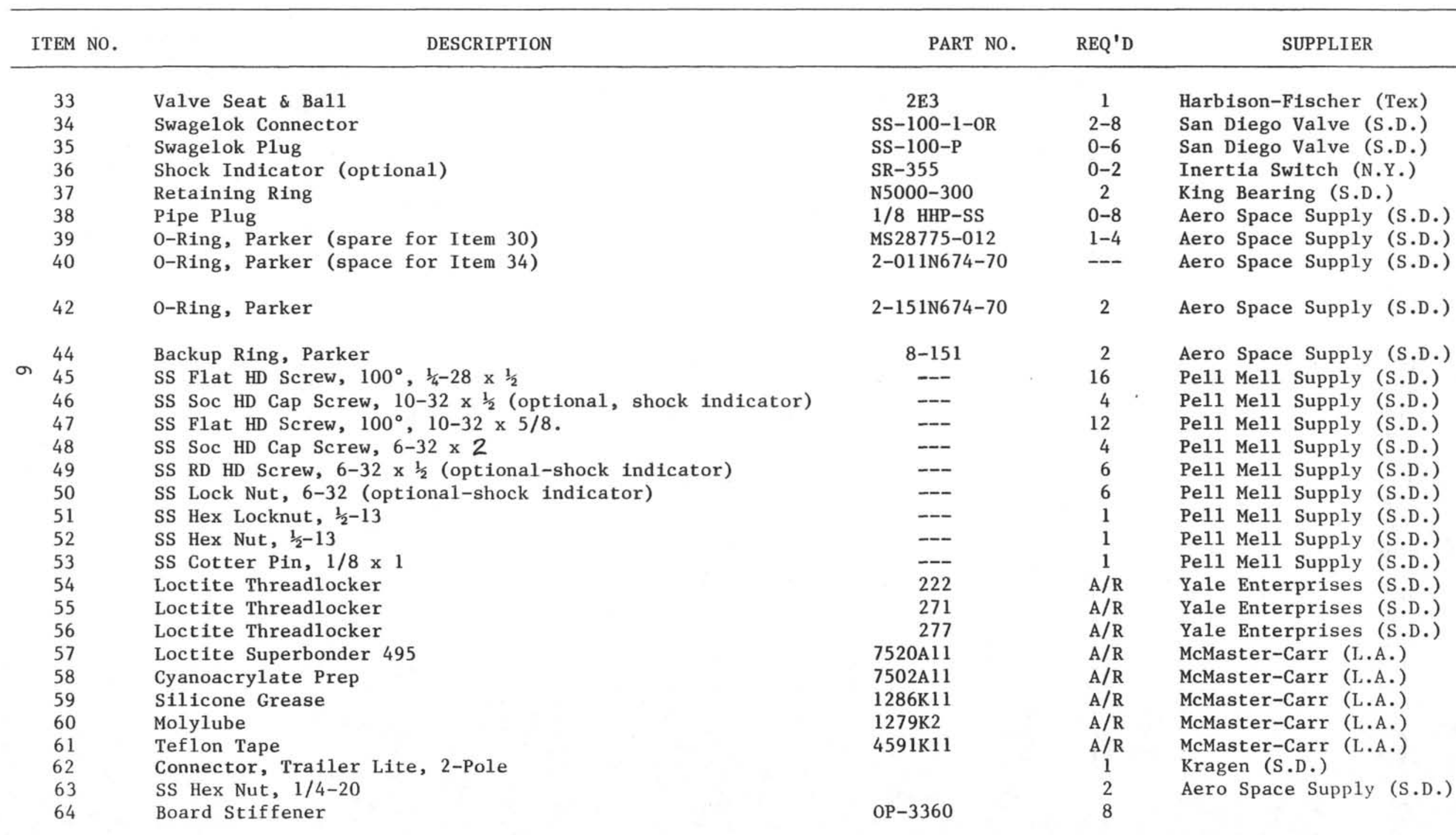


PARTS LIST - CORE BARREL INSTRUMENTATION, PRESSURE (OP3330) ELECTRONICS

\begin{tabular}{|c|c|c|c|c|c|}
\hline ITEM & No. & DESCRIPTION & & REQ'D & SUPPLIER \\
\hline 1 & RAM & & MB8167-70C & 12 & R.V. WEatherford (S.D.) \\
\hline 2 & $A-D$ & & $\mathrm{ADC} \mathrm{HC}-12 \mathrm{BMM}$ & 6 & Datel-Intersil (Santa Ana) \\
\hline 3 & IC & & CD $4051 \mathrm{BE}$ & 12 & Newark Electronics (S.D.) \\
\hline 4 & IC & & CD $4050 \mathrm{BE}$ & 7 & Newark Electronics (S.D.) \\
\hline 5 & IC & & CD $4518 \mathrm{BE}$ & 3 & Newark Electronics (S.D.) \\
\hline 6 & IC & & CD $4520 \mathrm{BE}$ & 3 & Newark Electronics (S.D.) \\
\hline 7 & IC & & $\mathrm{CD} 4059 \mathrm{AE}$ & 1 & Newark Electronics (S.D.) \\
\hline 8 & IC & & $\mathrm{CD} 4017 \mathrm{BE}$ & 4 & Newark Electronics (S.D.) \\
\hline 9 & IC & & $\mathrm{CD} 4045 \mathrm{BE}$ & 1 & Newark Electronics (S.D.) \\
\hline 10 & IC & & $\mathrm{CD} 4069 \mathrm{BE}$ & 2 & Newark Electronics (S.D.) \\
\hline 11 & IC & & $\mathrm{CD} 4053 \mathrm{BE}$ & 1 & Newark Electronics (S.D.) \\
\hline 12 & IC & & $\mathrm{CD} 4070 \mathrm{BE}$ & 2 & Newark Electronics (S.D.) \\
\hline$\checkmark 13$ & IC & & $\mathrm{CD} 4081 \mathrm{BE}$ & 2 & Newark Electronics (S.D.) \\
\hline 14 & IC & & $\mathrm{CD} 4022 \mathrm{BE}$ & 2 & Newark Electronics (S.D.) \\
\hline 15 & IC & & $\mathrm{CD} 4078 \mathrm{BE}$ & 3 & Newark Electronics (S.D.) \\
\hline 16 & IC & & $\mathrm{CD} 4013 \mathrm{BE}$ & 4 & Newark Electronics (S.D.) \\
\hline 17 & VR & & UA 7805 CKC & 1 & Newark Electronics (S.D.) \\
\hline 18 & VR & & UA 7810 CKC & 1 & Newark Electronics (S.D.) \\
\hline 19 & VR & & UA 7812 CKC & 1 & Newark Electronics (S.D.) \\
\hline 20 & Diode & & $1 \mathrm{~N} 914$ & 10 & Newark Electronics (S.D.) \\
\hline 21 & Crystal & & $76 \mathrm{~F} 829$ & 1 & Newark Electronics (S.D.) \\
\hline 22 & Dip Switch & & DYS8 & 1 & Newark Electronics (S.D.) \\
\hline 23 & Pot (Bournes) & & $13 F 3419$ & 12 & Newark Electronics (S.D.) \\
\hline 24 & CAP & & CK05BX104 & 13 & Newark Electronics (S.D.) \\
\hline 25 & CAP (Sprague) & & $17 \mathrm{~F} 2053$ & 6 & Newark Electronics (S.D.) \\
\hline 26 & CAP, Trim (Johanson) & & 9614 & 1 & Newark Electronics (S.D.) \\
\hline 27 & Header (A1pha) & & FCC 151-26 & 1 & Newark Electronics (S.D.) \\
\hline 28 & Push Button & & $13 F 3647$ & 1 & Newark Electronics (S.D.) \\
\hline 29 & CAP & & $14 \mathrm{~F} 1270$ & 1 & Newark Electronics (S.D.) \\
\hline 30 & CAP & & $14 \mathrm{~F} 1274$ & 1 & Newark Electronics (S.D.) \\
\hline 31 & CAP & & $14 \mathrm{~F} 1281$ & 1 & Newark Electronics (S.D.) \\
\hline 32 & CAP & & $13 \mathrm{~F} 5054$ & 1 & Newark Electronics (S.D.) \\
\hline 33 & Socket & & $38 \mathrm{~F} 1560$ & 1 & Newark Electronics (S.D.) \\
\hline
\end{tabular}




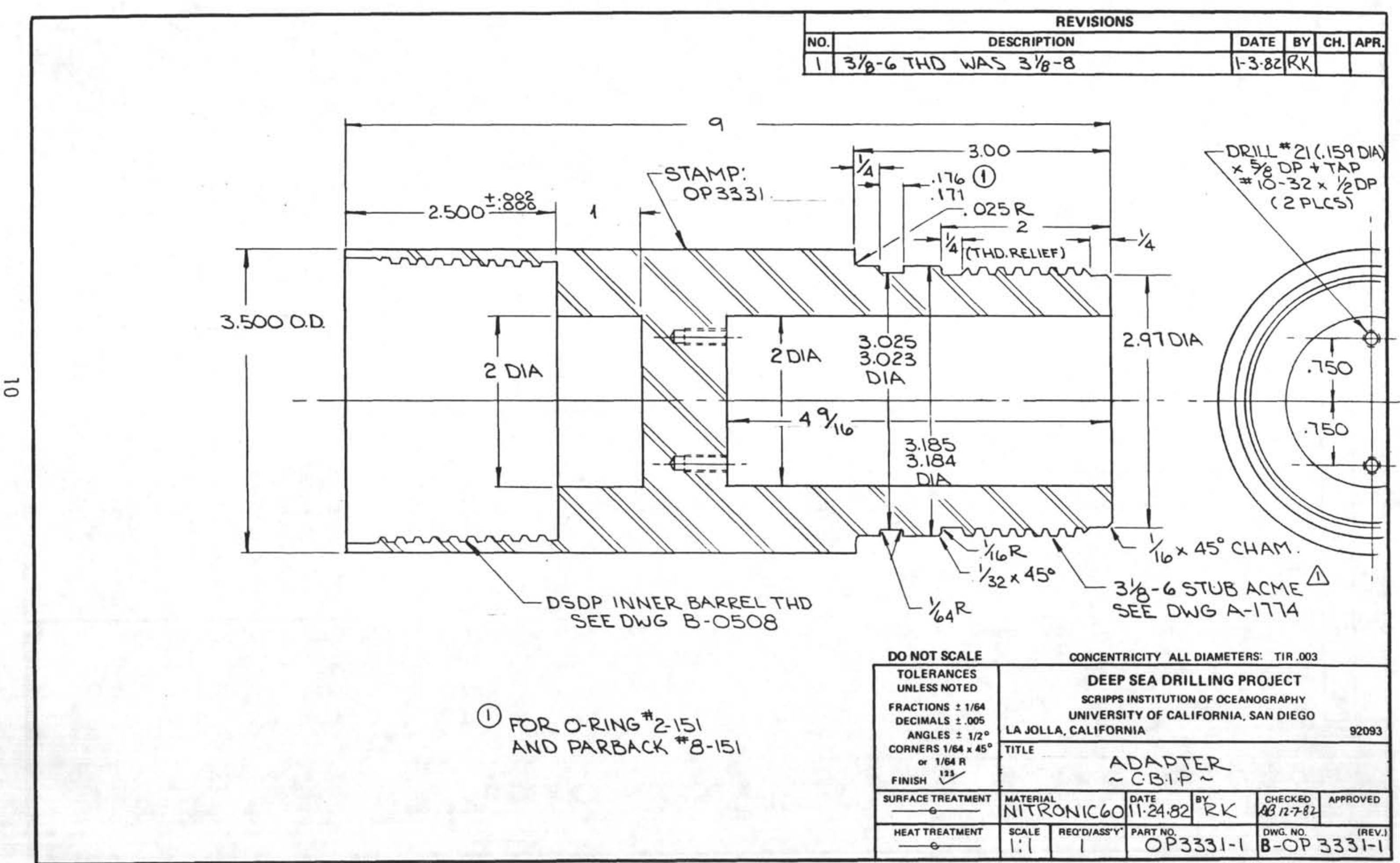




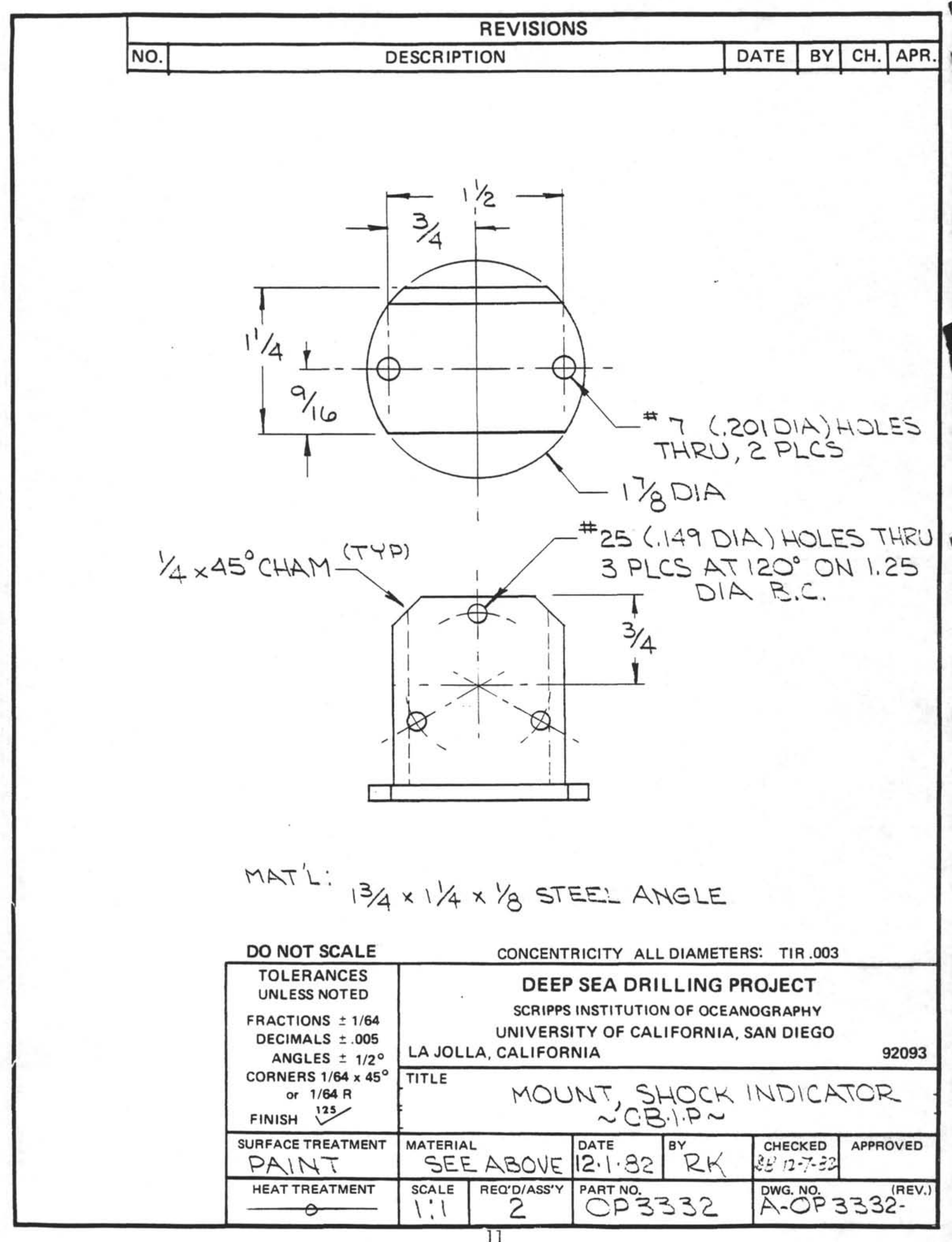




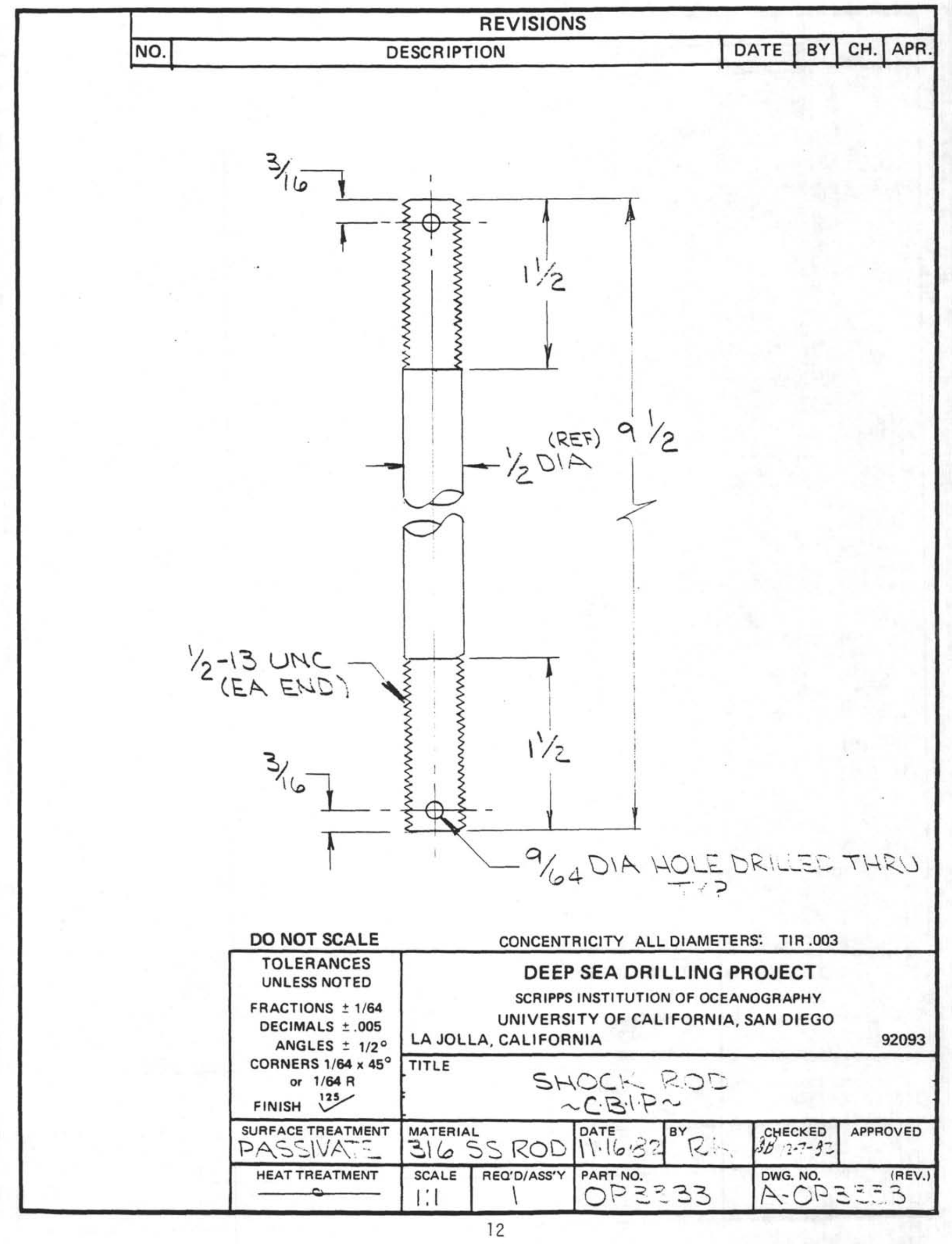




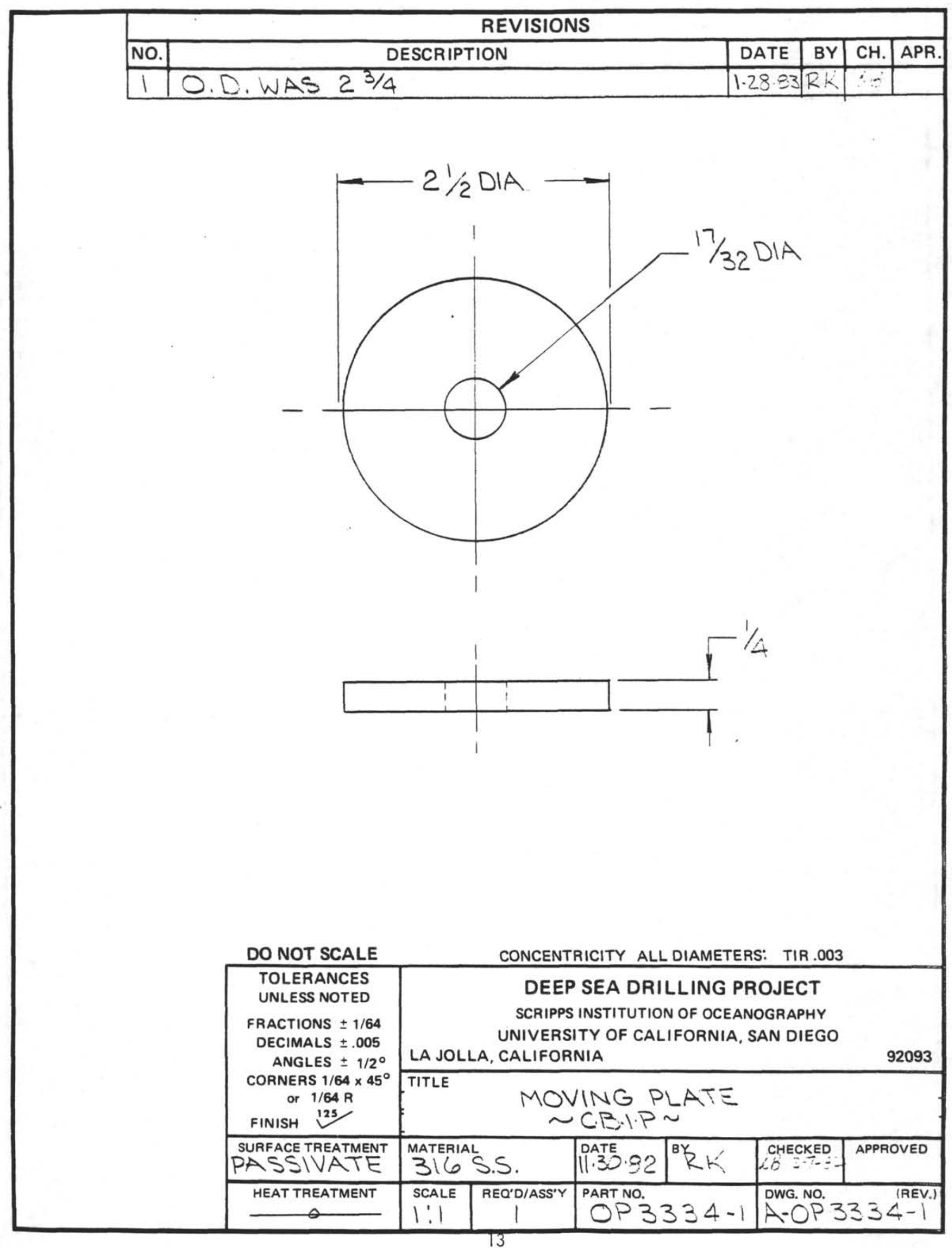




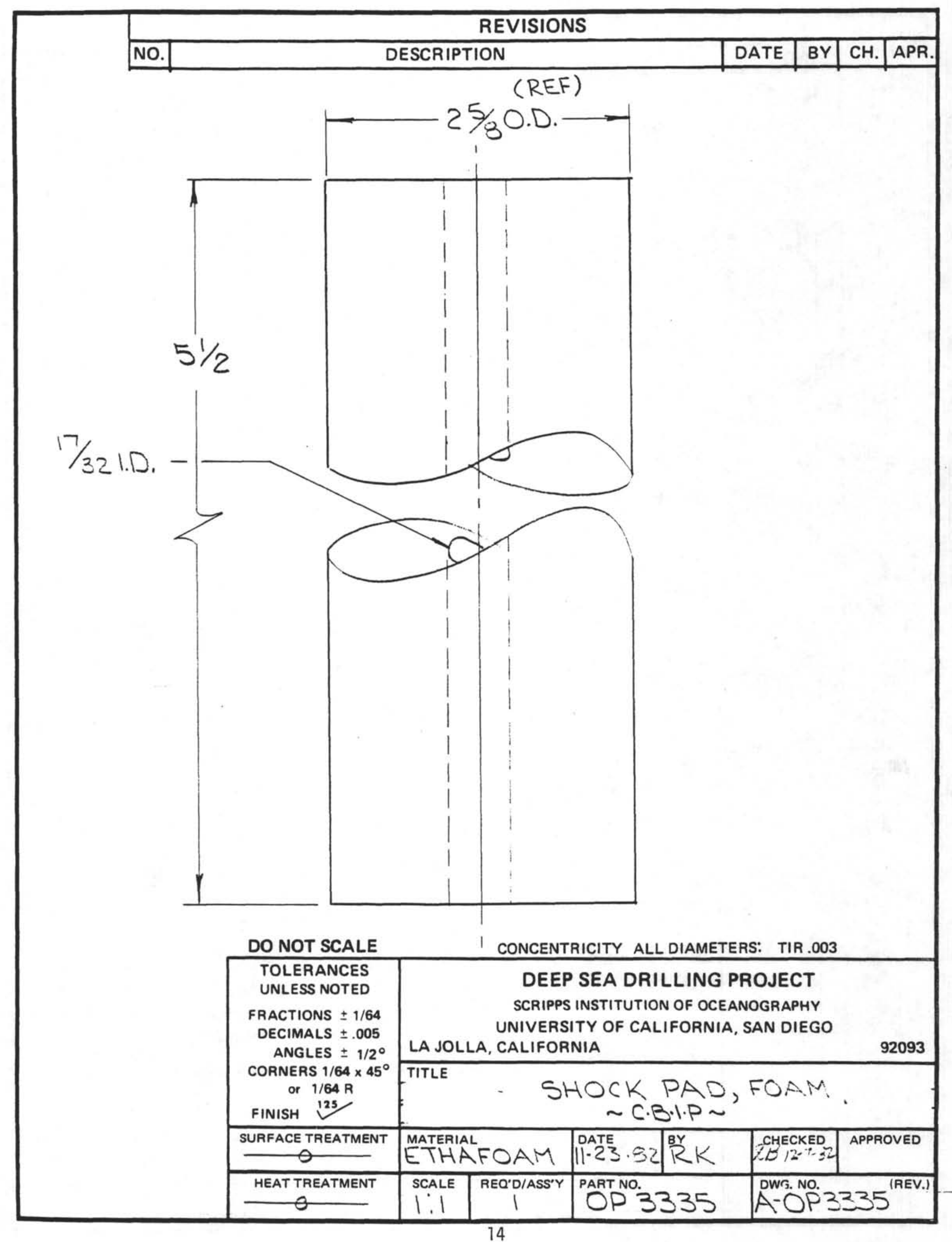




\begin{tabular}{|l|l|l|l|l|l|}
\hline NO. & DESCRIPTION & DATE & BY & CH. & APR. \\
\hline
\end{tabular}

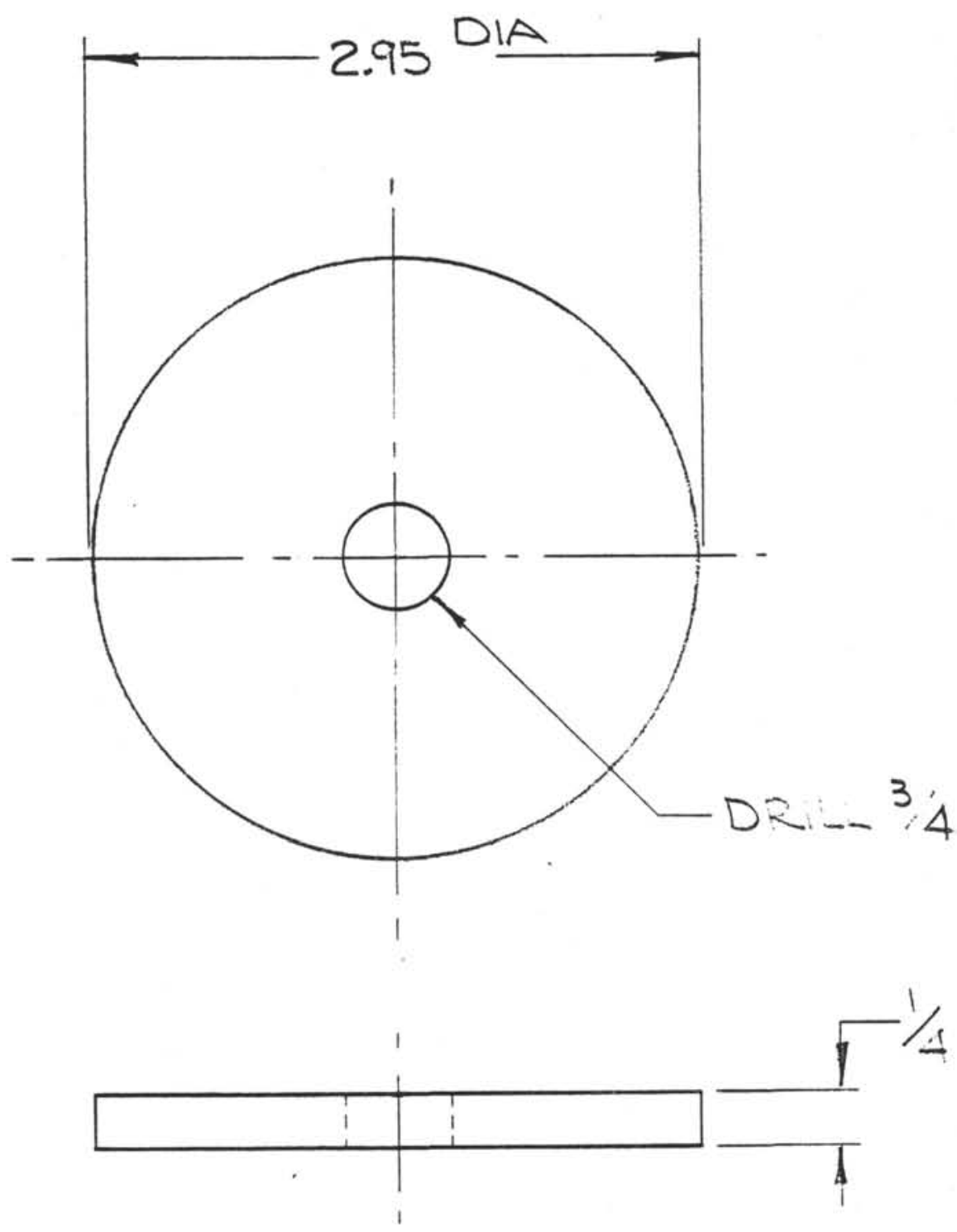

DO NOT SCALE TOLERANCES UNLESS NOTED

FRACTIONS $\pm 1 / 64$ DECIMALS \pm .005 ANGLES $\pm 1 / 2^{\circ}$ CORNERS $1 / 64 \times 45^{\circ}$ or $1 / 64 R$ FINISH 125 CONCENTRICITY ALL DIAMETERS: TIR .003

\section{DEEP SEA DRILLING PROJECT}

SCRIPPS INSTITUTION OF OCEANOGRAPHY UNIVERSITY OF CALIFORNIA, SAN DIEGO LA JOLLA, CALIFORNIA

TITLE

STOP PLATE $\sim C \cdot B \cdot P \sim$

SURFACE TREATMENT PASSIVATE

HEAT TREATMENT

O

MATERIAL

316 S.S.

DATE

$11.22 \cdot 32$ ${ }^{B Y} k^{\prime}$

1:1




\begin{tabular}{|l|l|l|l|l|l|}
\hline NO. & DESCRIPTION & DATE & BY & CH. & APR. \\
\hline
\end{tabular}

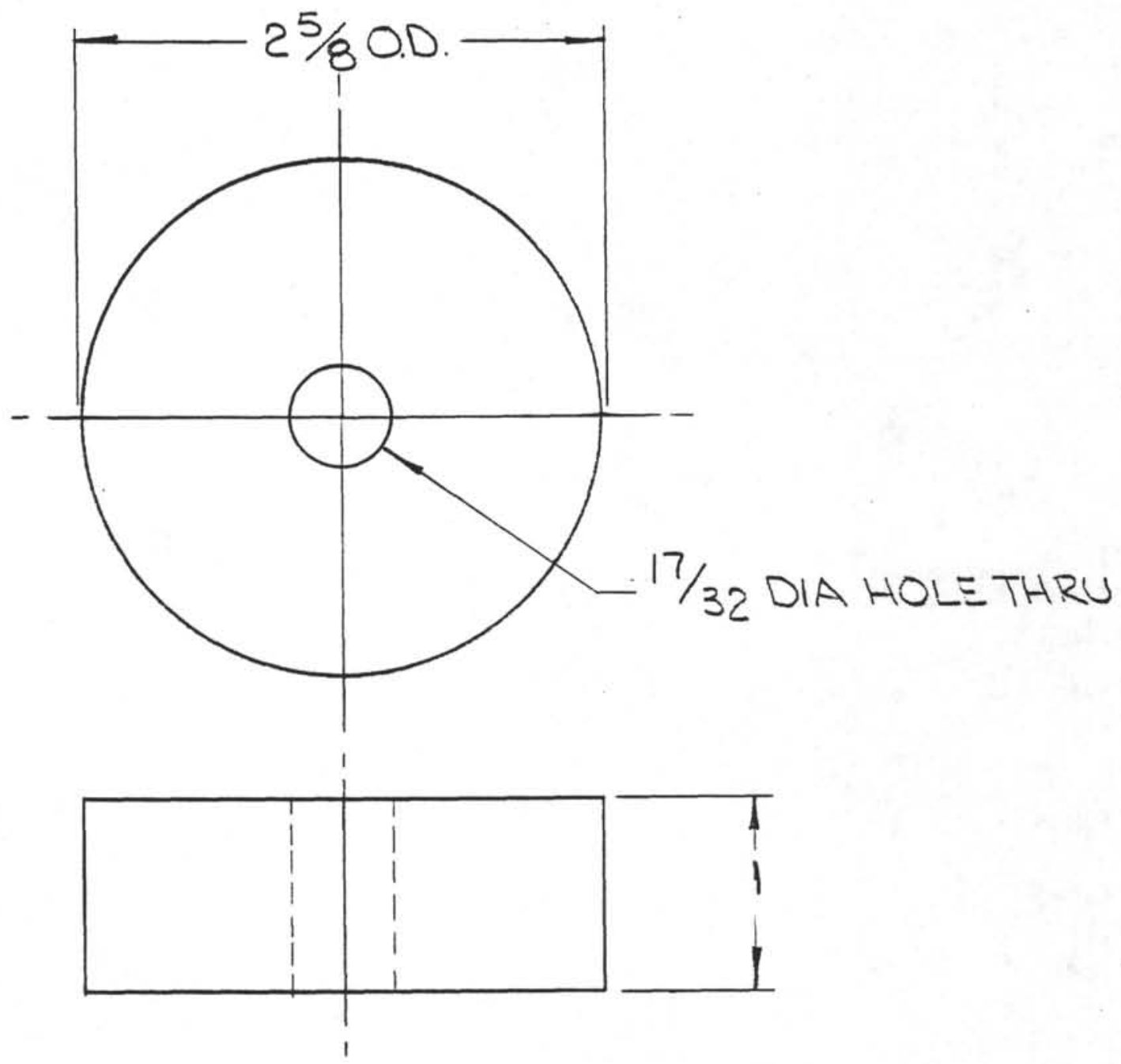

DO NOT SCALE TOLERANCES UNLESS NOTED

FRACTIONS $\pm 1 / 64$ DECIMALS \pm .005

ANGLES $\pm 1 / 2^{\circ}$ CORNERS $1 / 64 \times 45^{\circ}$ or $1 / 64 R$ FINISH 125 SURFACE TREATMENT

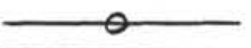
HEAT TREATMENT
CONCENTRICITY ALL DIAMETERS: TIR.003

DEEP SEA DRILLING PROJECT

SCRIPPS INSTITUTION OF OCEANOGRAPHY UNIVERSITY OF CALIFORNIA, SAN DIEGO

LA JOLLA, CALIFORNIA

REBOUND PAD $\sim$ C.B.I.P

DATE 1 BY 1 CHECKED

11.30 .82 RK

De is-7.

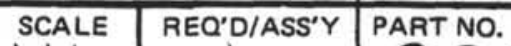

$1: 1$




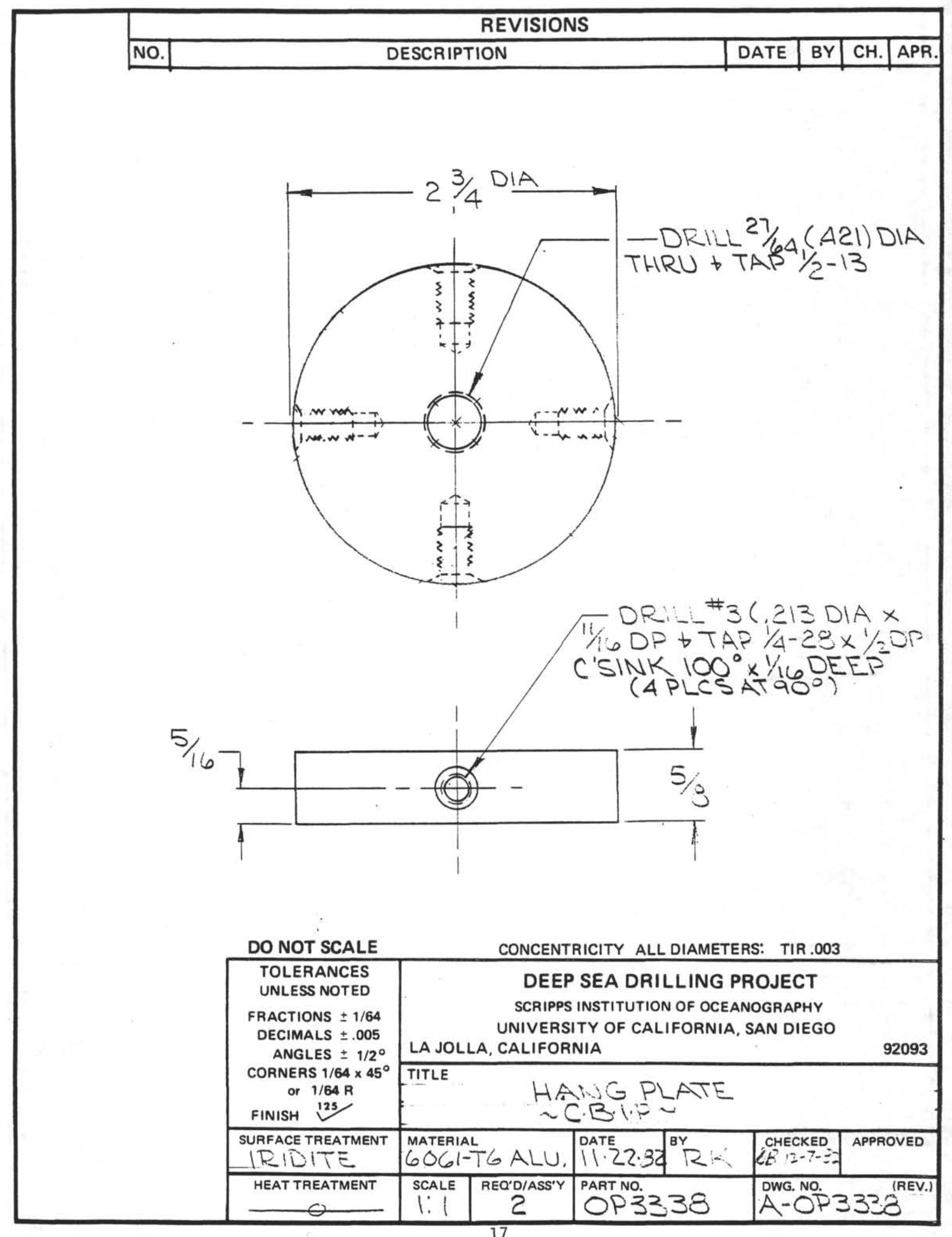




\begin{tabular}{|c|cc|c|c|c|c|}
\hline \multicolumn{3}{|c|}{ REVISIONS } & DATE & BY & CH. & APR. \\
\hline NO. & DESCRIPTION & 1.28 .83 & RK & $86^{3}$ & \\
\hline 1 & RE DESIGNED & 1.28 .83 & \\
\hline
\end{tabular}

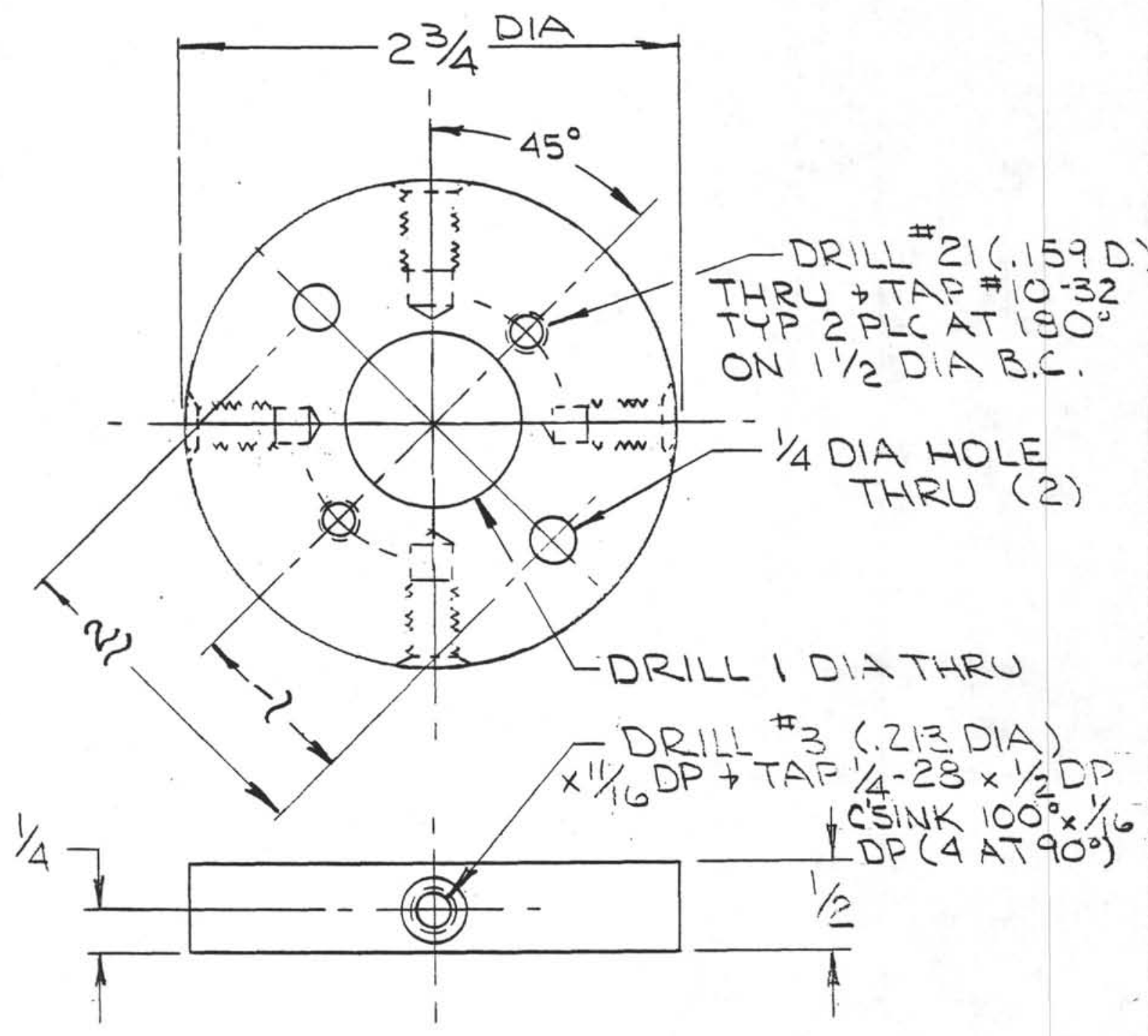

DO NOT SCALE TOLERANCES UNLESS NOTED

FRACTIONS $\pm 1 / 64$ DECIMALS \pm .005 ANGLES $\pm 1 / 2^{\circ}$ CORNERS $1 / 64 \times 45^{\circ}$ or $1 / 64 \mathrm{R}$ FINISH SURFACE TREATMENT IRIDITE HEAT TREATMENT 0

CONCENTRICITY ALL DIAMETERS: TIR .003

\section{DEEP SEA DRILLING PROJECT}

SCRIPPS INSTITUTION OF OCEANOGRAPHY UNIVERSITY OF CALIFORNIA, SAN DIEGO

LA JOLLA; CALIFORNIA

TITLE

PLATE, BATTERT $\sim C B I P \sim$

\begin{tabular}{|c|c|c|c|c|c|}
\hline OOC & 6 & $\begin{array}{l}\text { DATE } \\
11.23 .82\end{array}$ & ${ }^{B Y} R K$ & $\begin{array}{c}\text { CHECKED } \\
6^{\prime}: 2-7-3=1\end{array}$ & APPROVED \\
\hline $\begin{array}{l}\text { SCALE } \\
1 ; 1\end{array}$ & $\begin{array}{c}\text { REO'D/A } \\
3\end{array}$ & PART NO. & & $\begin{array}{l}\text { DWG. NO. } \\
A-O P\end{array}$ & $39-1$ \\
\hline
\end{tabular}




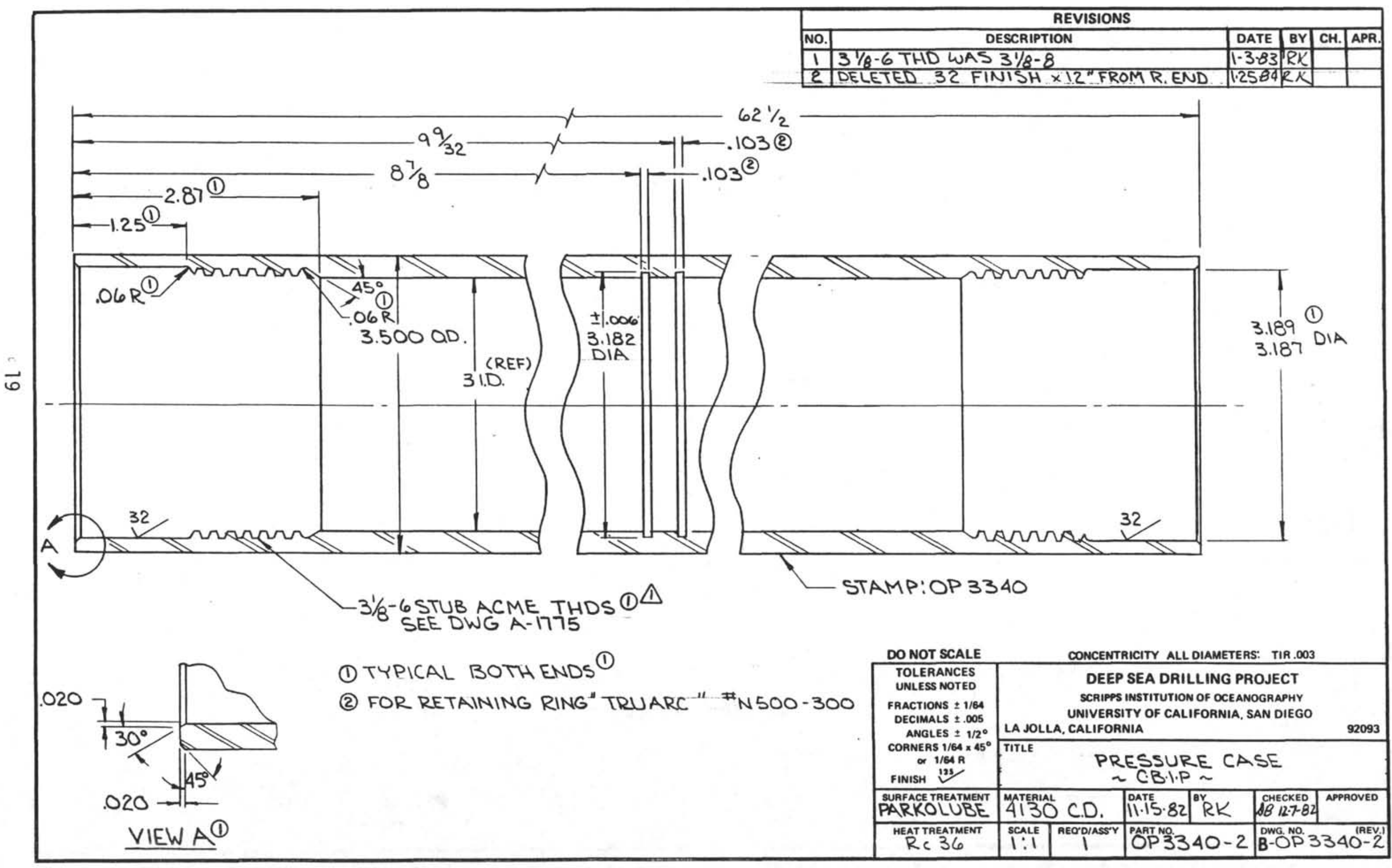




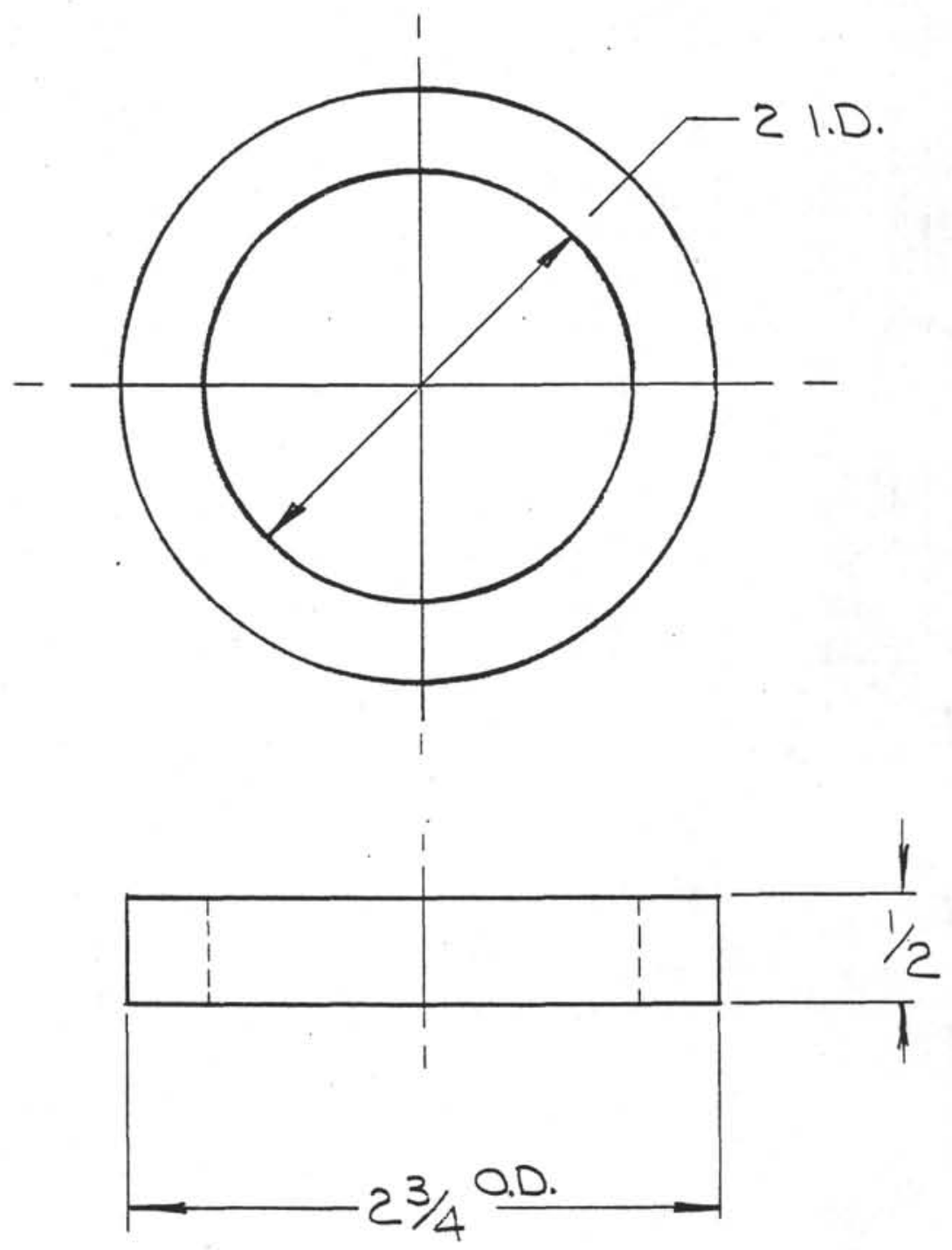

DO NOT SCALE

TOLERANCES

UNLESS NOTED

FRACTIONS $\pm 1 / 64$

DECIMALS \pm .000

ANGLES $\pm 1 / 2^{\circ}$

CORNERS $1 / 14$ × $46^{\circ}$

of Vten R

FINISH

128

SURFACE TREATMENT

HEAT TAEATMENT

HEAT TAEATIMENT
CONCENTRICITY ALL DIAMETERS: TIR.003

DEEP SEA DRILLING PROJECT

SCAIPPS INSTITUTION OF OCEANOGRAPHY

UNIVERSITY OF CALIFORNIA, SAN DIEGO

LA JOLLA, CALIFORNIA

92093

PLATE, INSULATOR $\sim$ C.B.I.P

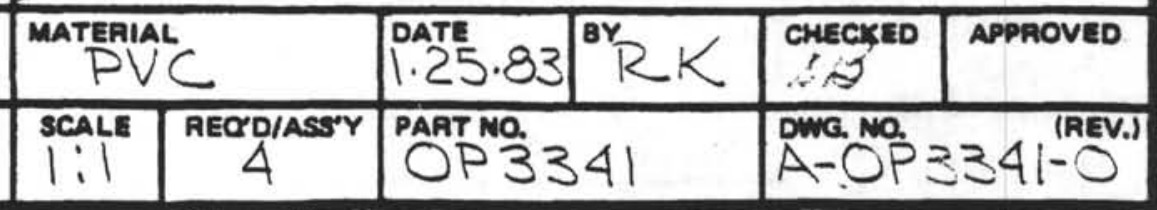




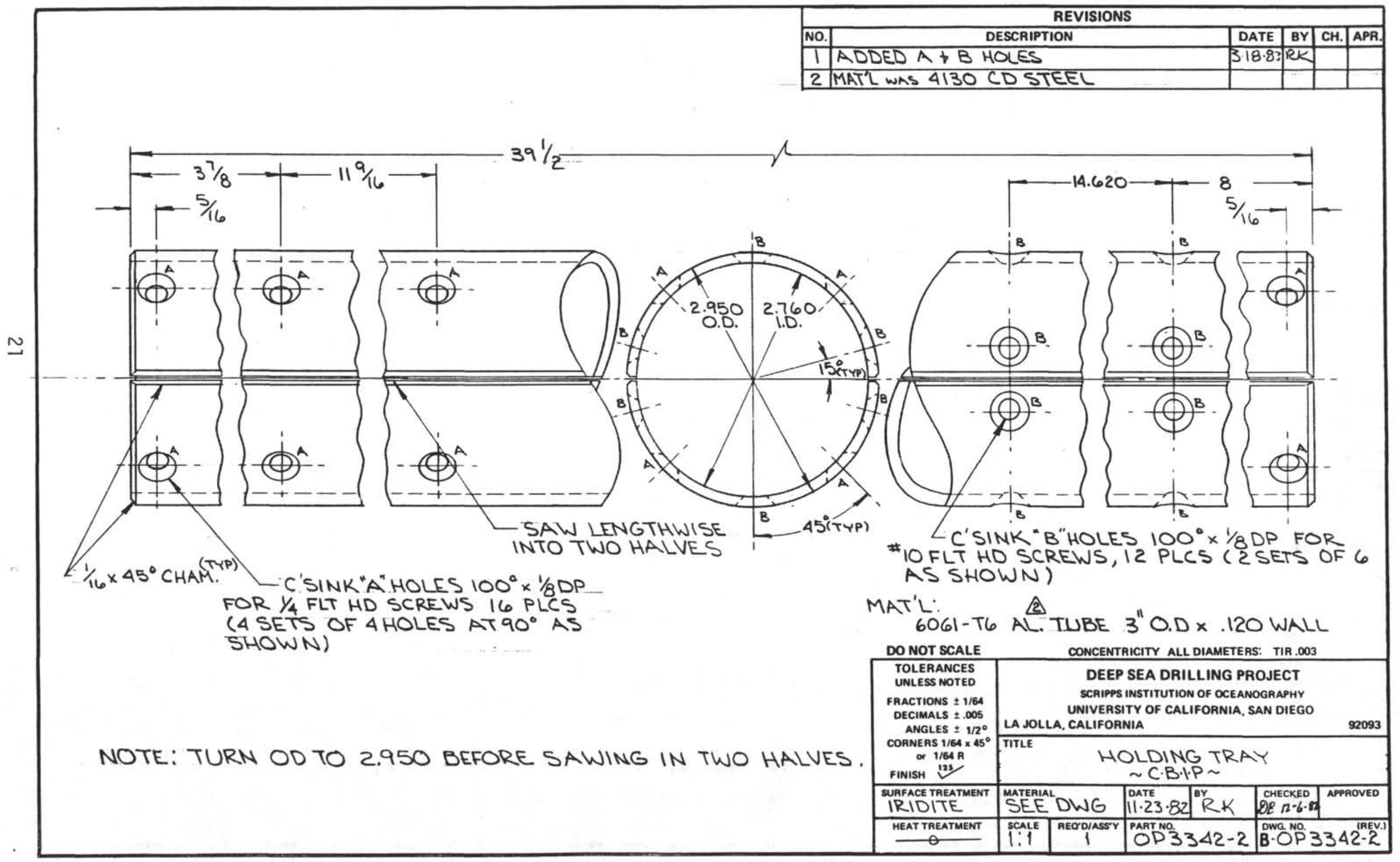




\begin{tabular}{|c|c|c|c|c|c|}
\hline \multicolumn{3}{|c|}{ REVISIONS } \\
\hline NO. & DESCRIPTION & DATE & BY & CH. & APR. \\
\hline 1 & 2.75 WAS 2 DIA. & & & & \\
\hline
\end{tabular}

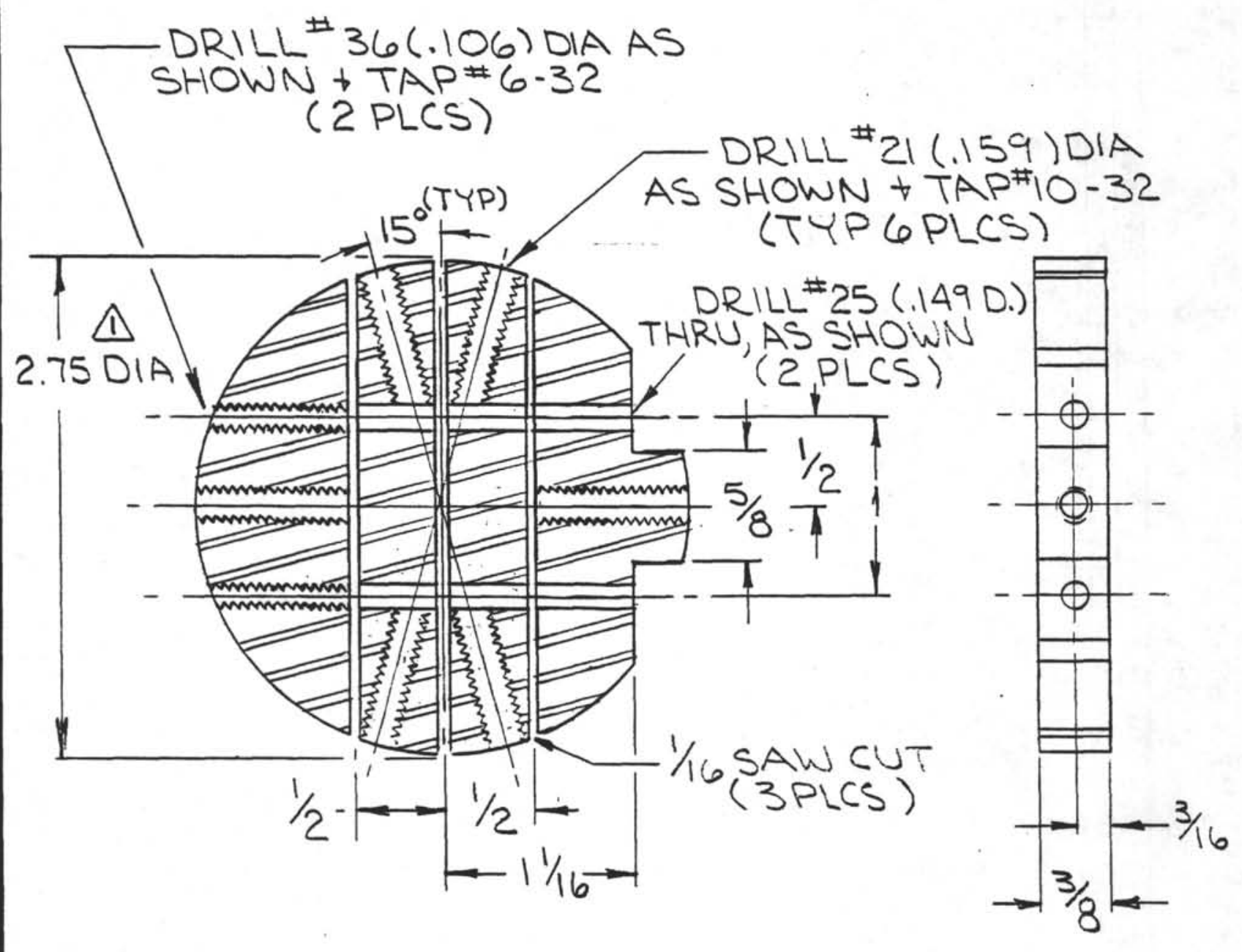

DO NOT SCALE

CONCENTRICITY ALL DIAMETERS: TIR. 003

\begin{tabular}{|c|}
\hline TOLERANCES \\
UNLESS NOTED \\
FRACTIONS $\pm 1 / 64$ \\
DECIMALS \pm .005 \\
ANGLES $\pm 1 / 2^{\circ}$ \\
CORNERS $1 / 64 \times 45^{\circ}$ \\
or $1 / 64 R$ \\
FINISH 125 \\
\hline SURFACE TREATMENT \\
IRID ITE \\
\hline HEAT TREATMENT \\
\hline
\end{tabular}

\section{DEEP SEA DRILLING PROJECT}

SCRIPPS INSTITUTION OF OCEANOGRAPHY UNIVERSITY OF CALIFORNIA, SAN DIEGO

LA JOLLA, CALIFORNIA

92093

ELECTROMICS PLATE $\sim C \cdot B l \cdot P \sim$ 
- DRILL .172 DIA THRU,

4 PLCS AT $90^{\circ}$ ON $115 / 8$

DIA B.C. (SEE DETAIL A BELOW)

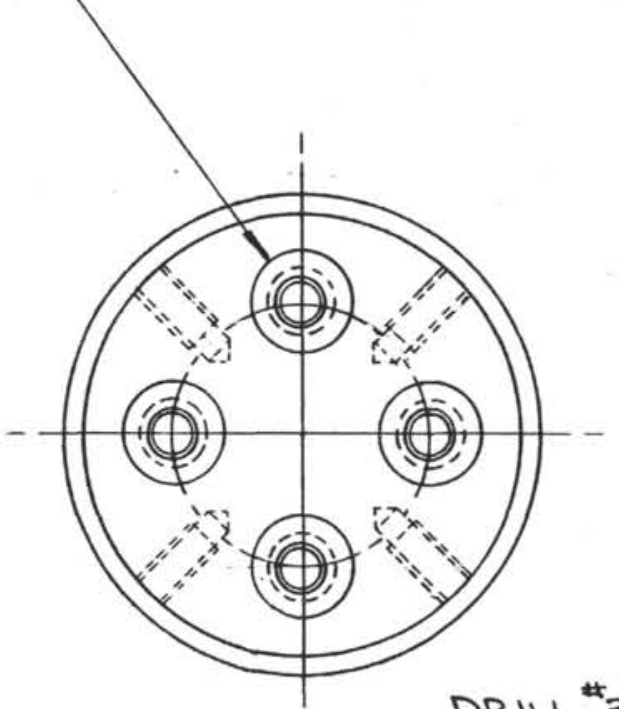

DRILL "3(.213D.) $\times 5 / 8 D P+T A P 1 / 4-28 \times 1 / 2$

DEEP, TYP 4 PLCS@90
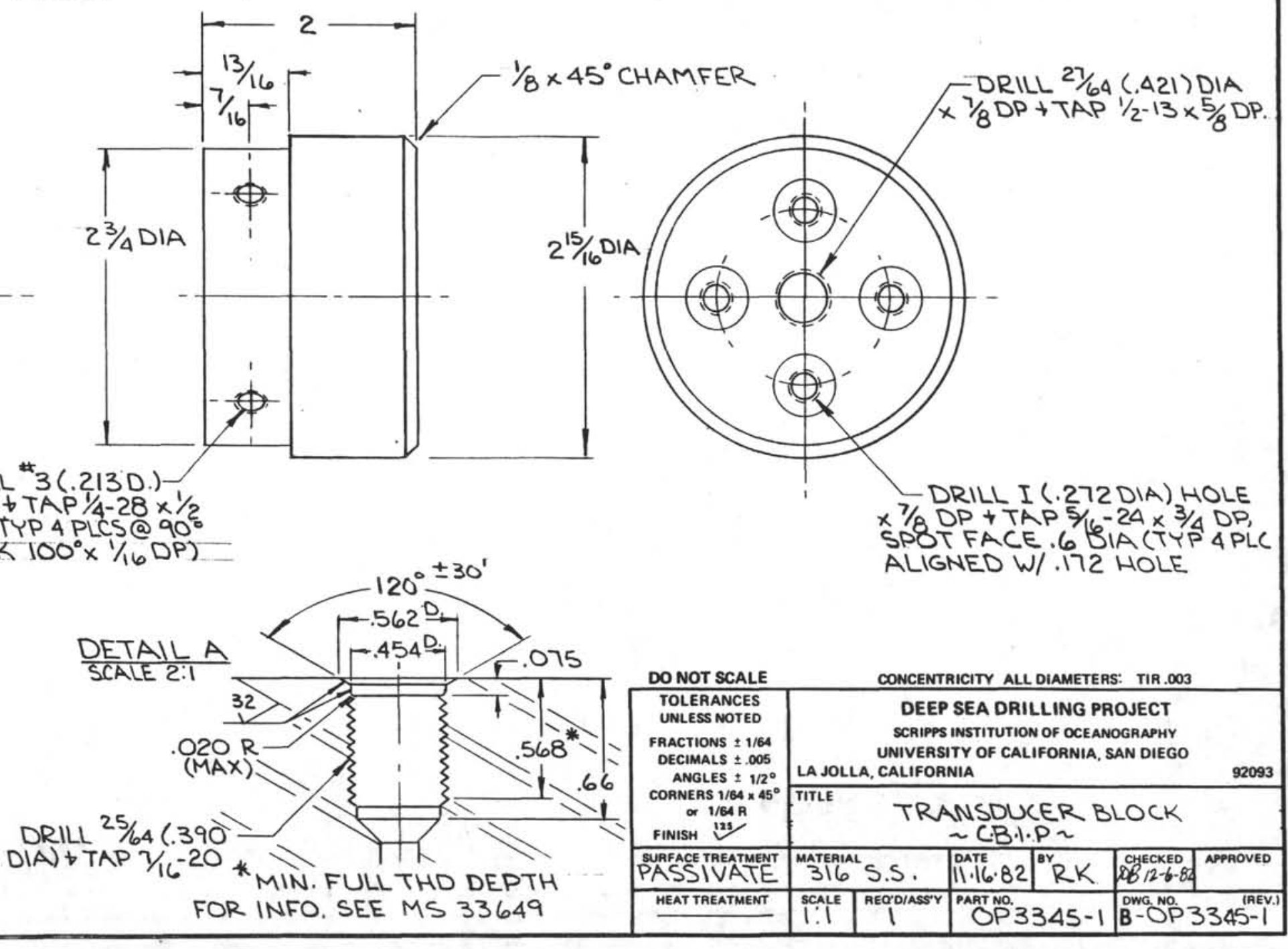


\begin{tabular}{|l|l|l|l|l|l|}
\hline NO. & DESCRIPTION & DATE & BY & CH. & APR. \\
\hline
\end{tabular}

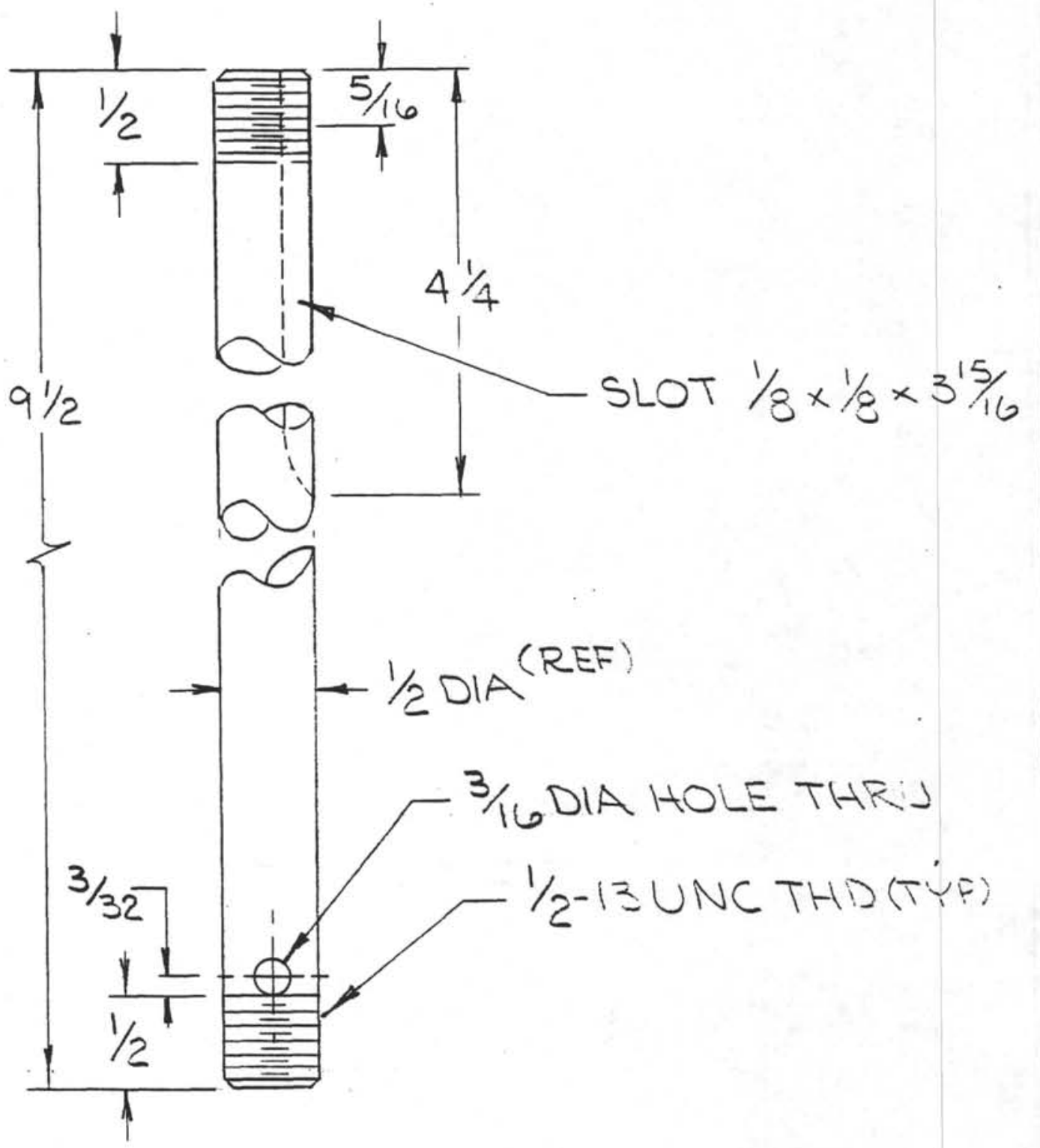

DO NOT SCALE

\begin{tabular}{|c|}
\hline TOLERANCES \\
UNLESS NOTED \\
FRACTIONS $\pm 1 / 64$ \\
DECIMALS \pm .005 \\
ANGLES $\pm 1 / 2^{\circ}$ \\
CORNERS $1 / 64 \times 45^{\circ}$ \\
or $1 / 64 R$ \\
FINISH VI \\
\hline SURFACE TREATMENT \\
PA $\leq$ IVATE \\
\hline HEAT TREATMENT \\
\hline O
\end{tabular}

CONCENTRICITY ALL DIAMETERS: TIR.003

\section{DEEP SEA DRILLING PROJECT}

SCRIPPS INSTITUTION OF OCEANOGRAPHY UNIVERSITY OF CALIFORNIA, SAN DIEGO

LA JOLLA, CALIFORNIA TITLE

TORQUE ROD $\sim C \cdot B \cdot I \cdot \bar{P} \sim$ MATERIAL 316 S.S.

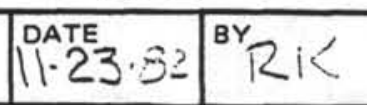
CHECKED APPROVED \begin{tabular}{l|l|l}
\hline SCALE & REQ'D/ASS'Y & PART NO.
\end{tabular} \begin{tabular}{ll|l}
$1: 1$ & 1 & OP3346
\end{tabular} DWG. NO. A.OP 3346 


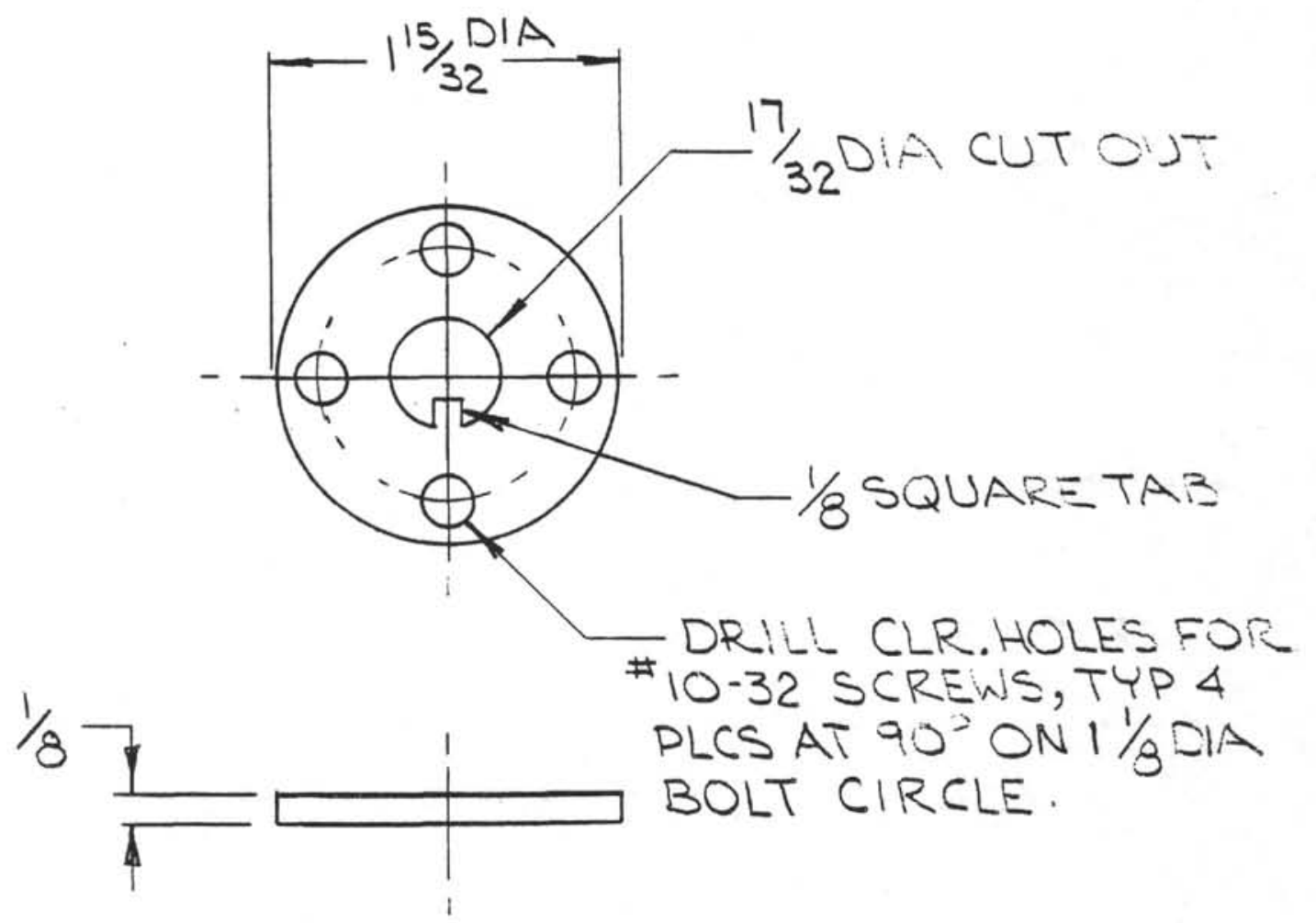

DO NOT SCALE TOLERANCES UNLESS NOTED

FRACTIONS $\pm 1 / 64$ DECIMALS \pm .005

ANGLES $\pm 1 / 2^{\circ}$ CORNERS $1 / 64 \times 45^{\circ}$ or $1 / 64 \mathrm{R}$ FINISH 125 SURFACE TREATMENT PASSIVATE HEAT TREATMENT $\theta$
CONCENTRICITY ALL DIAMETERS: TIR.003

\section{DEEP SEA DRILLING PROJECT}

SCRIPPS INSTITUTION OF OCEANOGRAPHY UNIVERSITY OF CALIFORNIA, SAN DIEGO LA JOLLA, CALIFORNIA 92093

PLATE, TORQUE ROD $\sim C \cdot B \cdot$. .P MATERIAL 3165.5 \begin{tabular}{|l|l|l|}
\hline DATE & BY & CHECKED \\
\hline
\end{tabular} DOB :2-7- $=$ APPROVED

SCALE $\mid$ REQ'D/ASS'Y PART NO.
1:1 DWG. NO.

A.OF 3347 


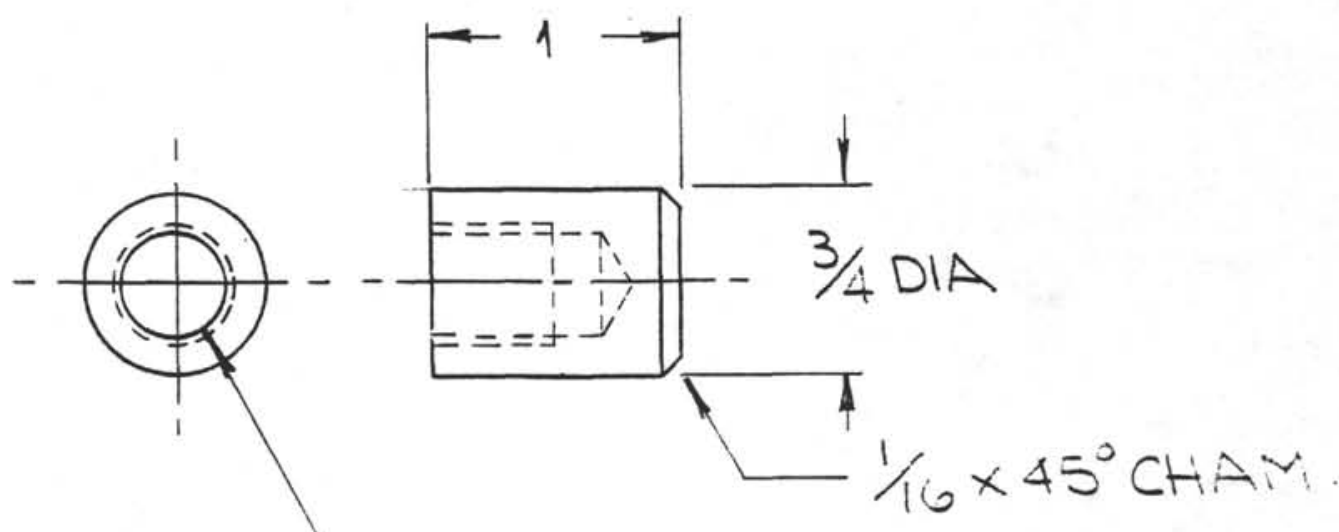

$$
\begin{aligned}
& 11 \text { DRIL } 27 / 64(, 422 \text { DIA }) \times \\
& 11 / 16 D P+T A P 1 / 2-13 \times 1 / 2 D P
\end{aligned}
$$

DO NOT SCALE TOLERANCES UNLESS NOTED

FRACTIONS $\pm 1 / 64$ DECIMALS \pm .005

ANGLES $\pm 1 / 2^{\circ}$ CORNERS $1 / 64 \times 45^{\circ}$ or $1 / 64 \mathrm{R}$ FINISH 125

T1T

LA JOL

DEEP SEA DRILLING PROJECT
SCRIPPS INSTITUTION OF OCEANOGRAPHY
UNIVERSITY OF CALIFORNIA, SAN DIEGO

UNIVERSITY OF CALIFORNIA, SAN DIEGO

CAP, TORQUE ROT

\begin{tabular}{|c|c|c|c|c|c|c|}
\hline $\begin{array}{l}\text { SURFACE TREATMENT } \\
\text { PASSIVATIE }\end{array}$ & $\begin{array}{l}\text { MATERIA } \\
316\end{array}$ & S.S & 11.22 .84 & 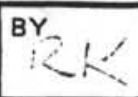 & $\begin{array}{c}\text { CHECKED } \\
C B \quad 12-7-27=1\end{array}$ & APPROVED \\
\hline $\begin{array}{c}\text { HEAT TREATMENT } \\
0\end{array}$ & SCALE & $\begin{array}{l}\text { REQ'D/ASS'Y } \\
\qquad\end{array}$ & PART NO. & & DWG. & $A 3^{\prime \prime}$ \\
\hline
\end{tabular}
$\approx C B . P \sim$ 


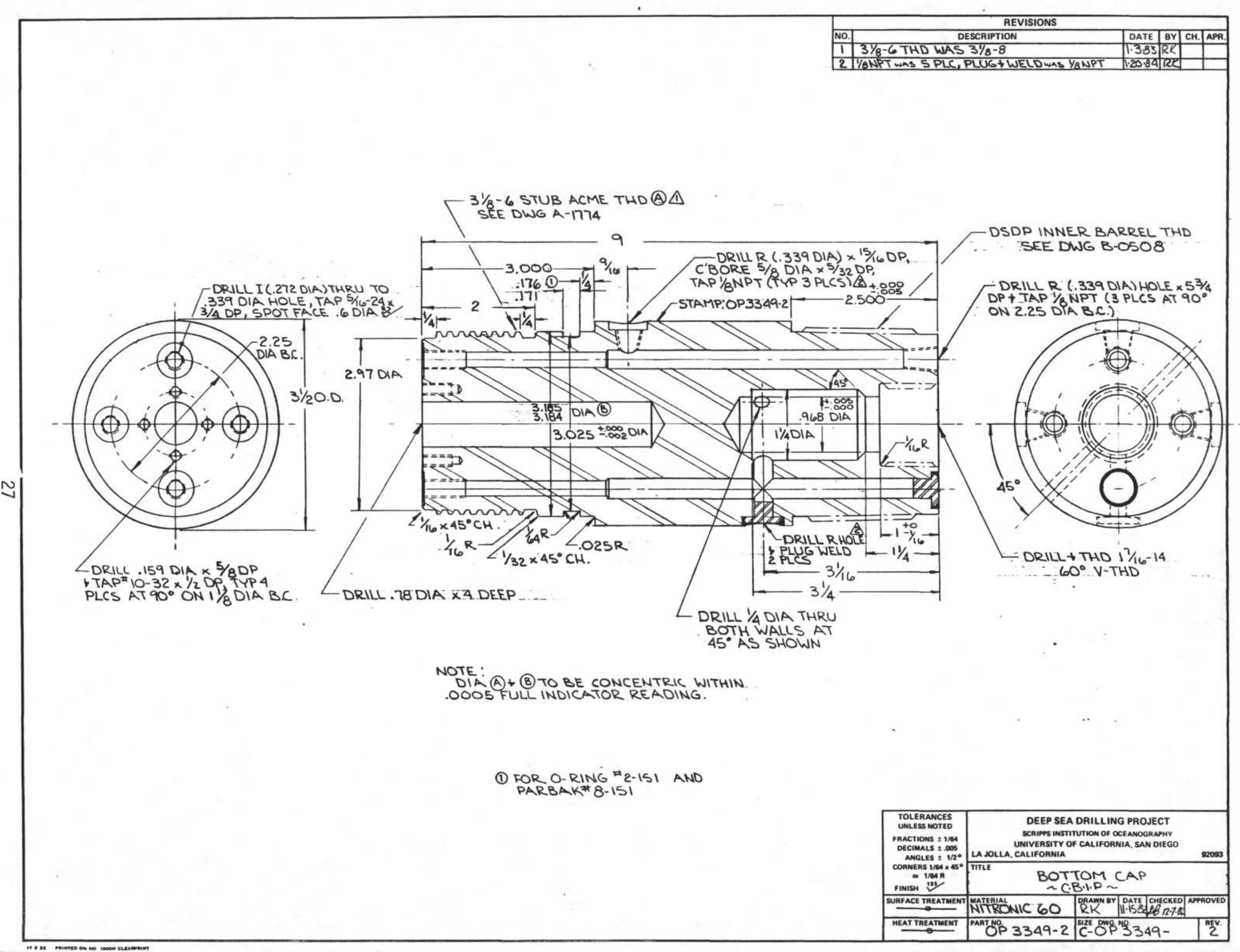




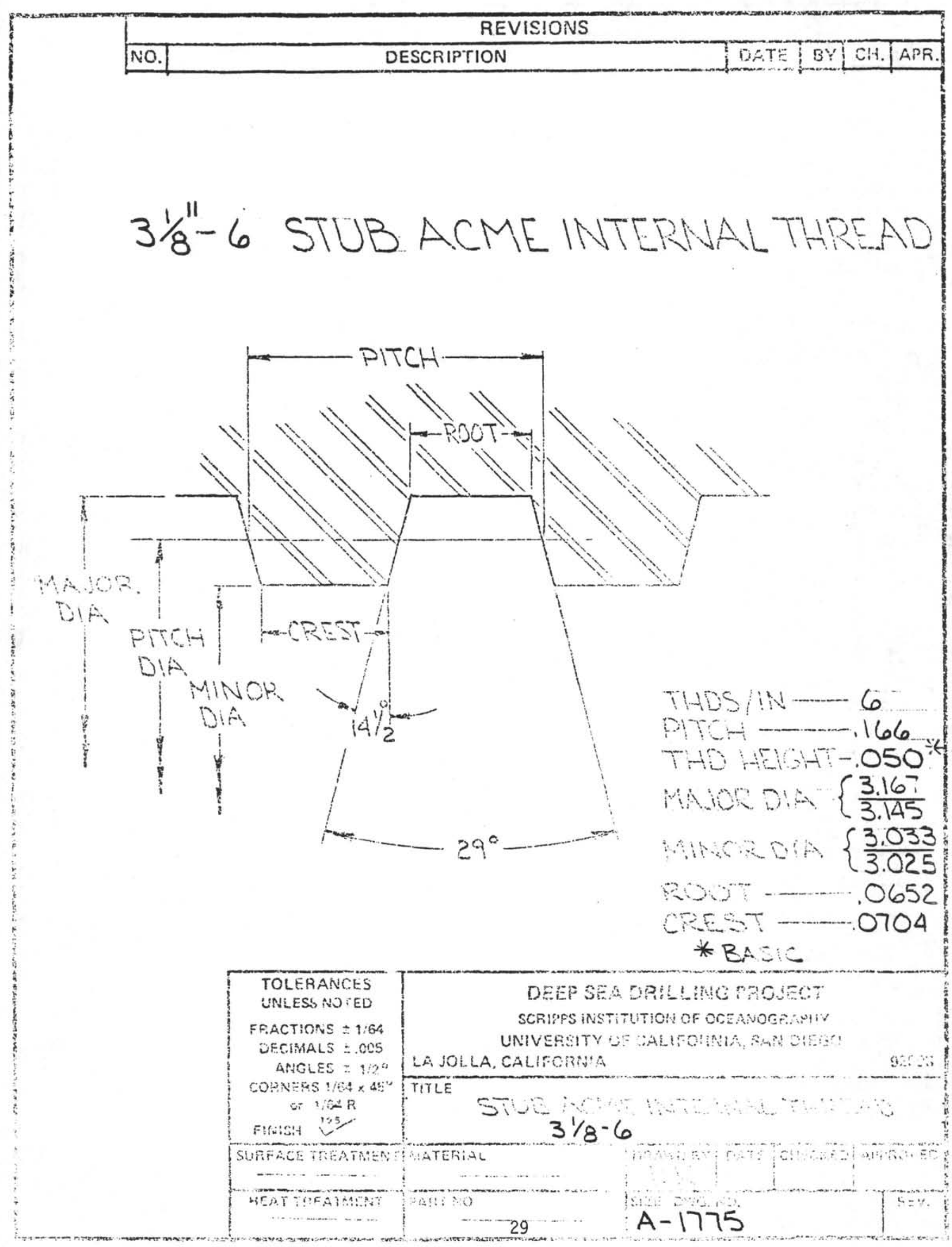




\begin{tabular}{|l|l|l|l|l|l|}
\hline NO. & DESCRIPTION & DATE & BY & CH. & APR. \\
\hline
\end{tabular}

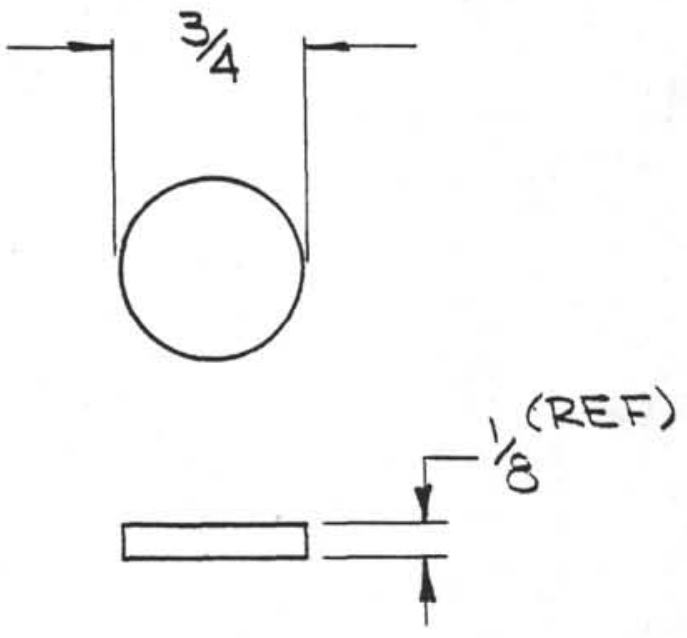

DO NOT SCALE TOLERANCES UNLESS NOTED

FRACTIONS $\pm 1 / 64$

DECIMALS \pm .005

ANGLES $\pm 1 / 2^{\circ}$ CORNERS $1 / 64 \times 45^{\circ}$

FINISH or $1 / 64 \mathrm{R}$

SURFACE TREATMENT
MATERIAL

MATS SHEET

$1: 1$
CONCENTRICITY ALL DIAMETERS: TIR.003

\section{DEEP SEA DRILLING PROJECT}

SCRIPPS INSTITUTION OF OCEANOGRAPHY UNIVERSITY OF CALIFORNIA, SAN DIEGO

LA JOLLA, CALIFORNIA

SHOCK PAD, RUEEER $\sim C \cdot B \cdot I \cdot P \sim$

\begin{tabular}{|l|l|l|}
\hline DATE & BY & CHECKED
\end{tabular}

APPROVED 


\begin{tabular}{|l|l|l|l|l|l|}
\hline NO. & DESCRIPTION & DATE & BY & CH. & APR. \\
\hline
\end{tabular}

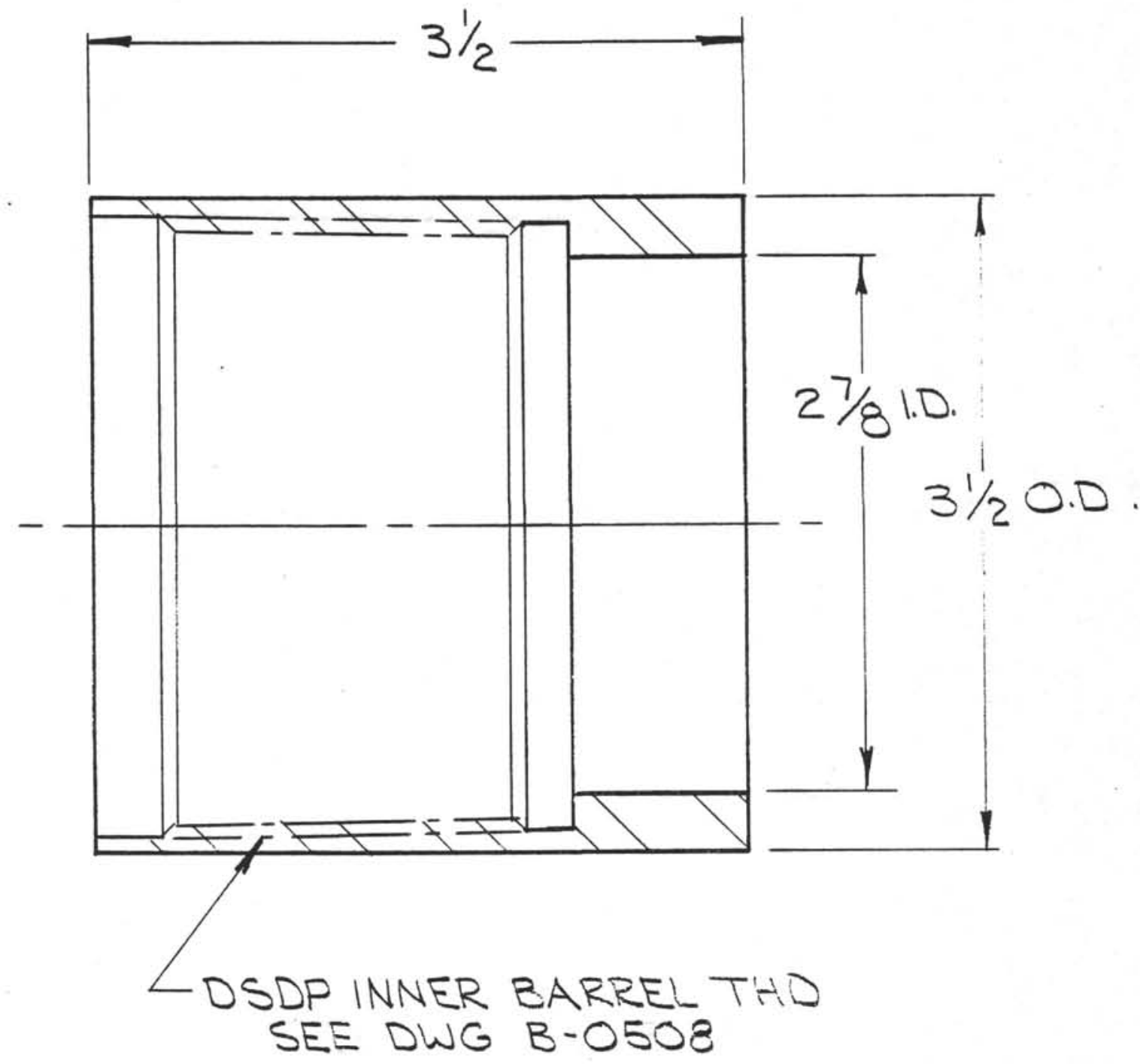

DO NOT SCALE TOLERANCES UNLESS NOTED

FRACTIONS $\pm 1 / 64$ DECIMALS \pm .005

ANGLES $\pm 1 / 2^{\circ}$ CORNERS $1 / 64 \times 45^{\circ}$ or $1 / 64 R$ FINISH 125 SURFACE TREATMENT PARKOLUBE
CONCENTRICITY ALL DIAMETERS: TIR.003

\section{DEEP SEA DRILLING PROJECT}

SCRIPPS INSTITUTION OF OCEANOGRAPHY UNIVERSITY OF CALIFORNIA, SAN DIEGO LA JOLLA, CALIFORNIA 92093

\begin{tabular}{|c|c|c|c|c|c|}
\hline \multicolumn{5}{|l|}{ TITLE } & . \\
\hline $\begin{array}{l}\text { MATERI } \\
413\end{array}$ & C.D. & DATE & RY K & $\begin{array}{l}\text { CHECKED } \\
\qquad / 3\end{array}$ & APPROVED \\
\hline $\begin{array}{l}\text { SCALE } \\
1: 1\end{array}$ & $\begin{array}{c}2^{R E O^{\prime} D / A S S^{\prime} Y} \\
2^{2}\end{array}$ & PART NO. & & DWG. NO. & $2=1$ \\
\hline
\end{tabular}




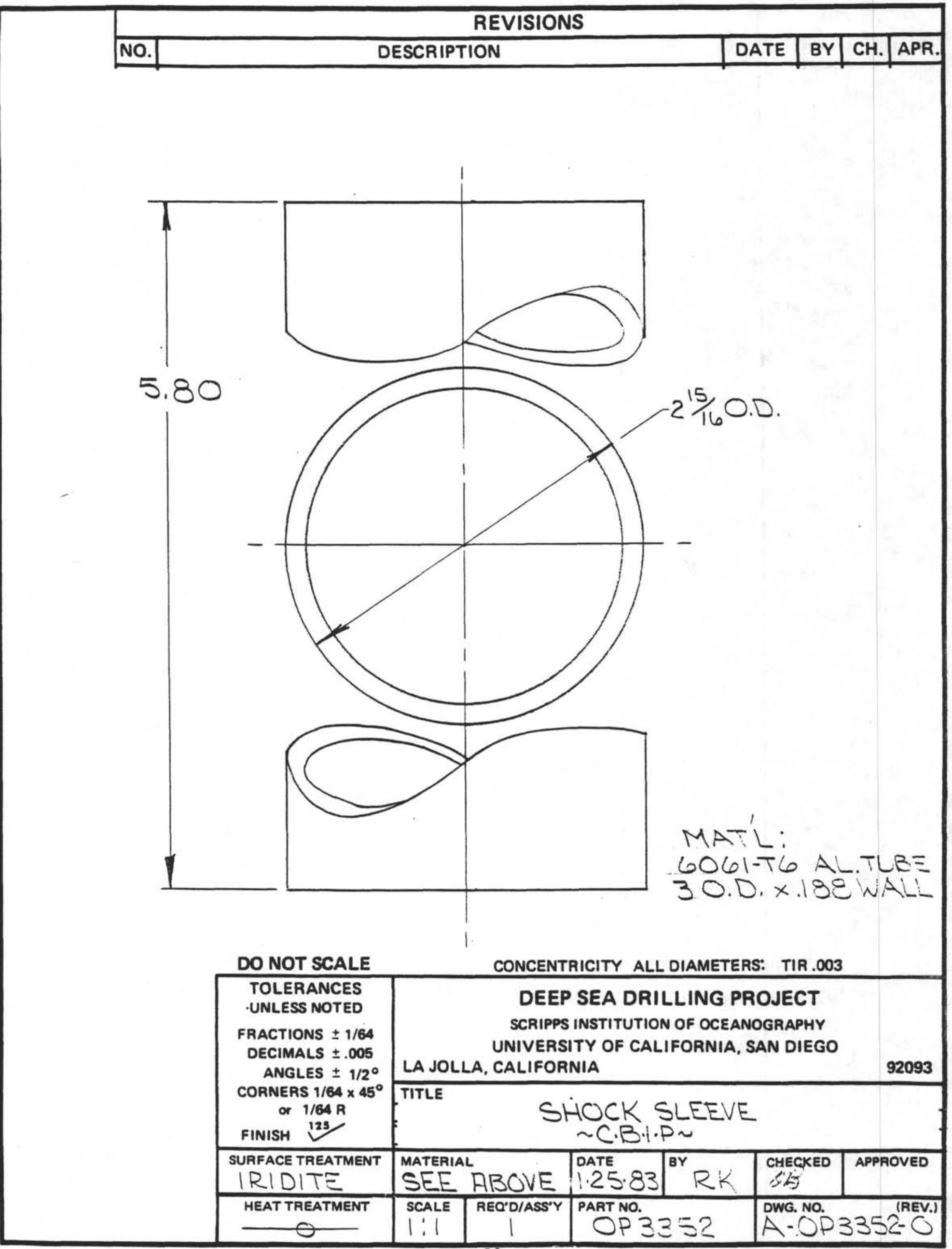




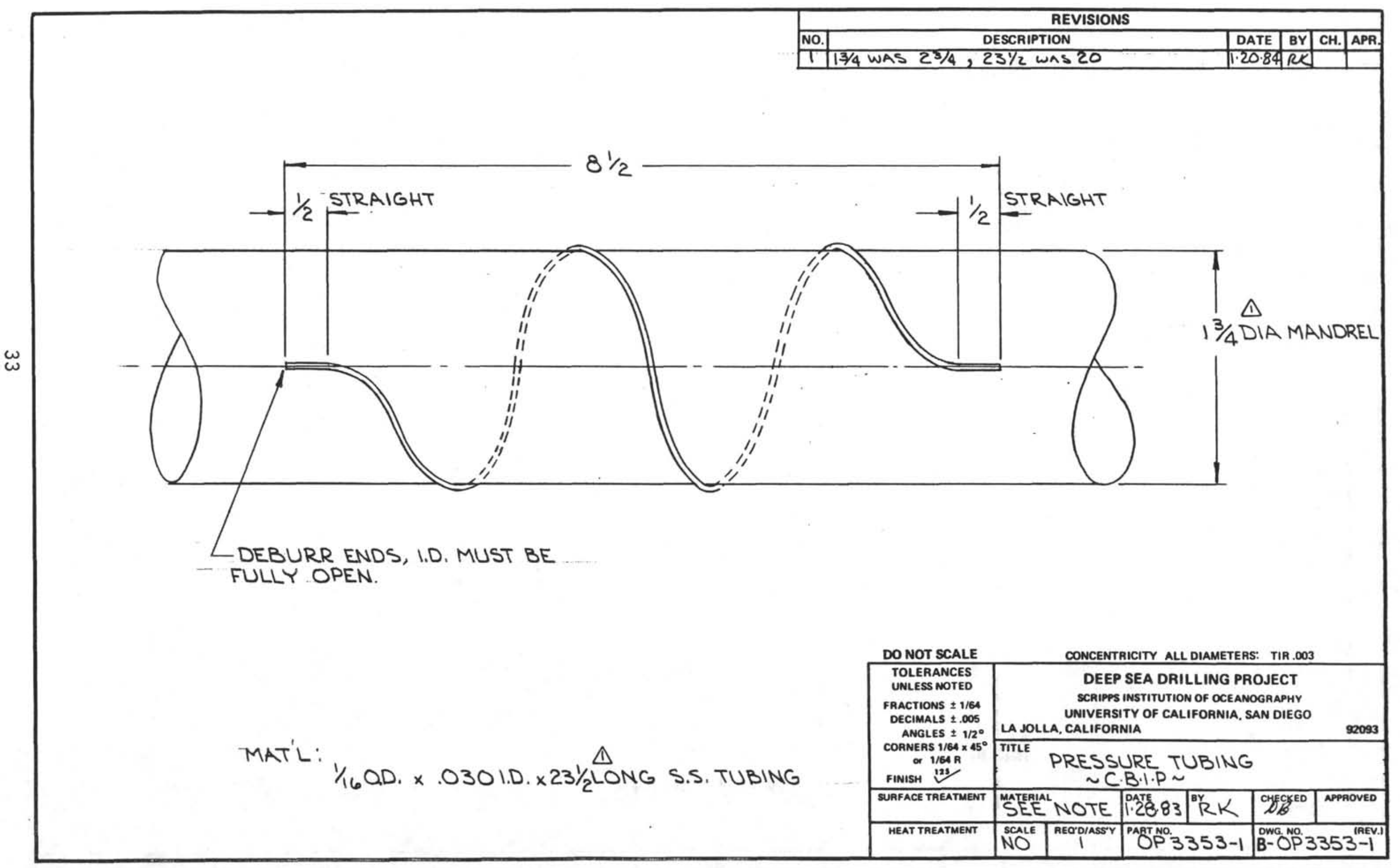


COMPONENTS AND BOARDS TO BE ARRANGED NOT TO EXCEED 2" DIAMETER WHEN ASSEMBLED
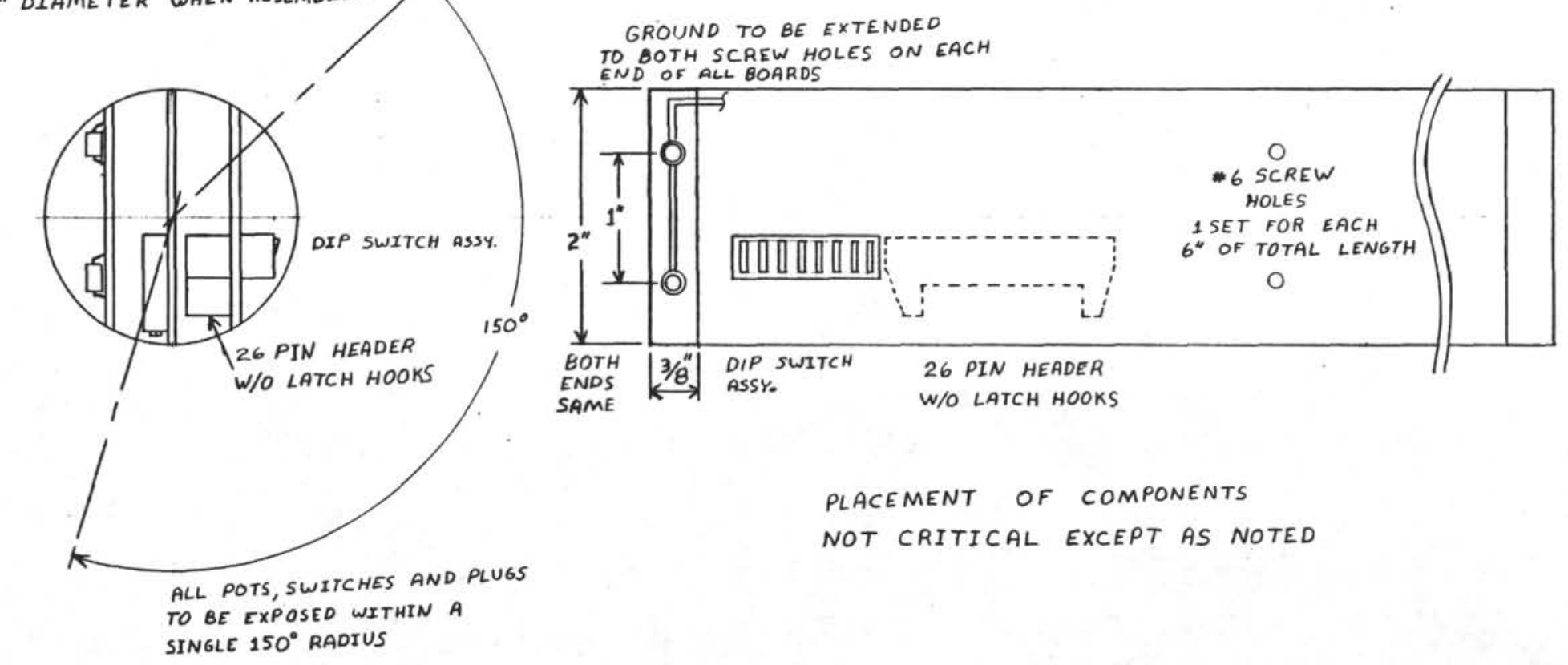

NUMBER OF BOARDS NOT FIXED AT 3

\begin{tabular}{|c|c|c|c|c|c|c|}
\hline \multirow{4}{*}{ 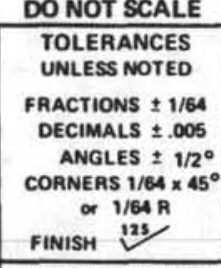 } & \multirow{2}{*}{\multicolumn{6}{|c|}{ CONCENTRICITY ALL DIAMETERS: TIR .003 }} \\
\hline & \multirow{2}{*}{\multicolumn{5}{|c|}{$\begin{array}{l}\text { DEEP SEA DRILLING PROJECT } \\
\text { SCRIPPS INSTITUTION OF OCEANOGRAPHY } \\
\text { UNIVERSTYY OF CALIFORNIA, SAN DIEGO } \\
\text { LA JOLLLA, CALIFORNIA }\end{array}$}} & \\
\hline & & & & & & 92093 \\
\hline & \multicolumn{6}{|c|}{ PITLE P.BOARD ASS'Y } \\
\hline SURFACE TREATMENT & \multicolumn{2}{|c|}{ MATERIAL } & DATE & $18 \mathrm{By}$ & CHECKED & APPROVED \\
\hline HEAT TREATMENT & SCALE & REQOOASSYY & PAATN & 3354 & \begin{tabular}{|l} 
DWG No- \\
B-OP,
\end{tabular} & $334^{\text {IAEV. }}$ \\
\hline
\end{tabular}




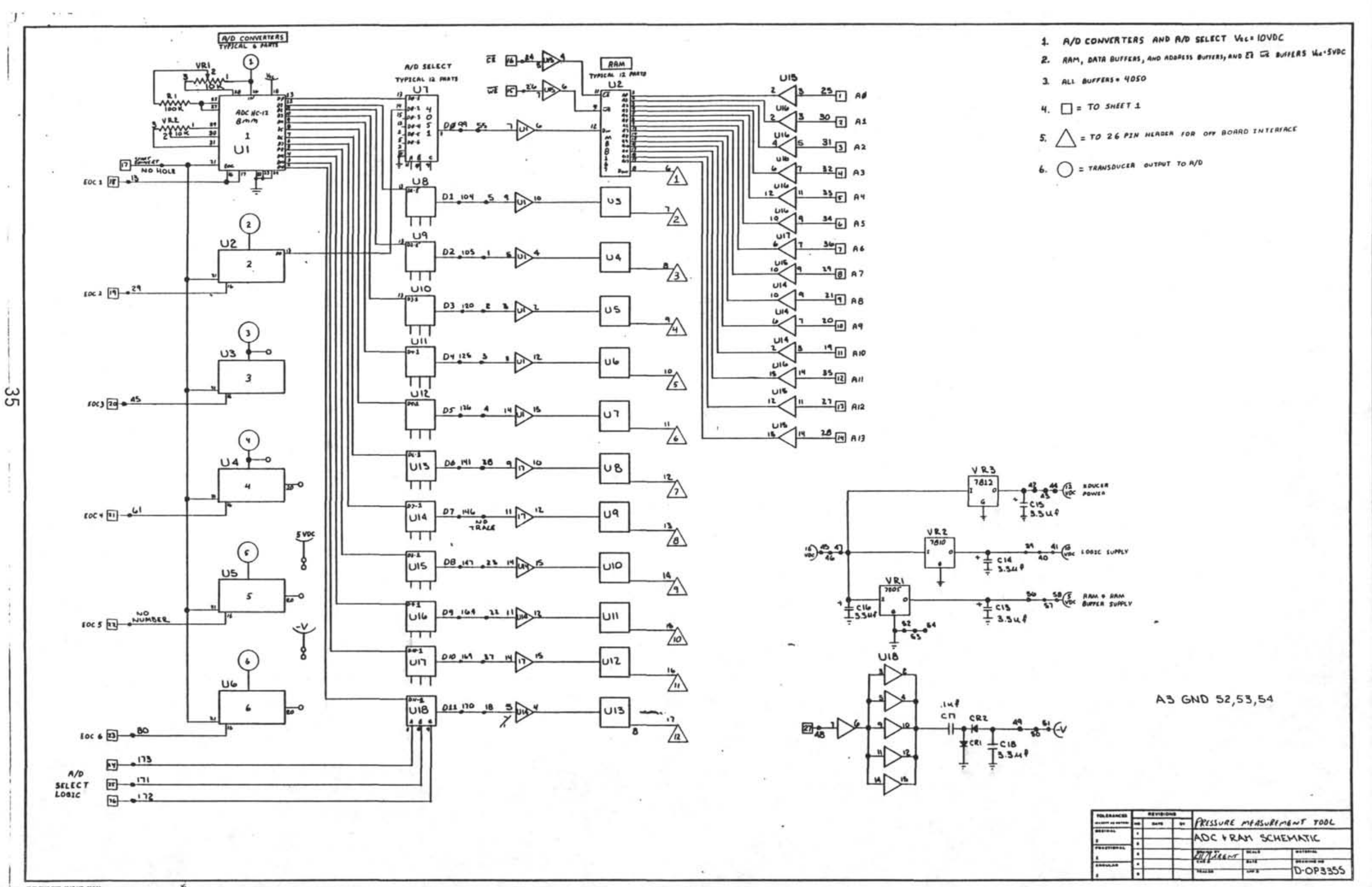




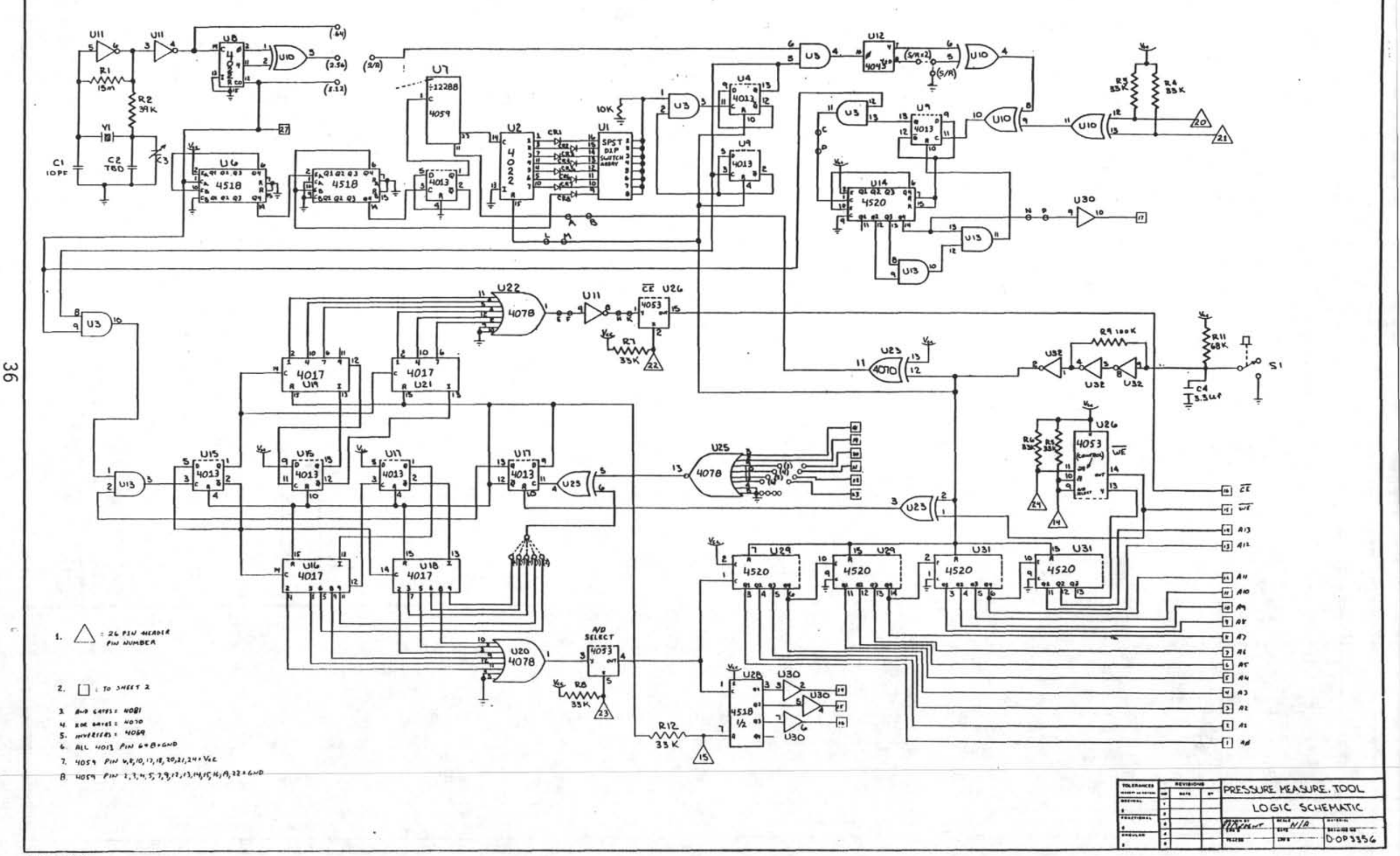




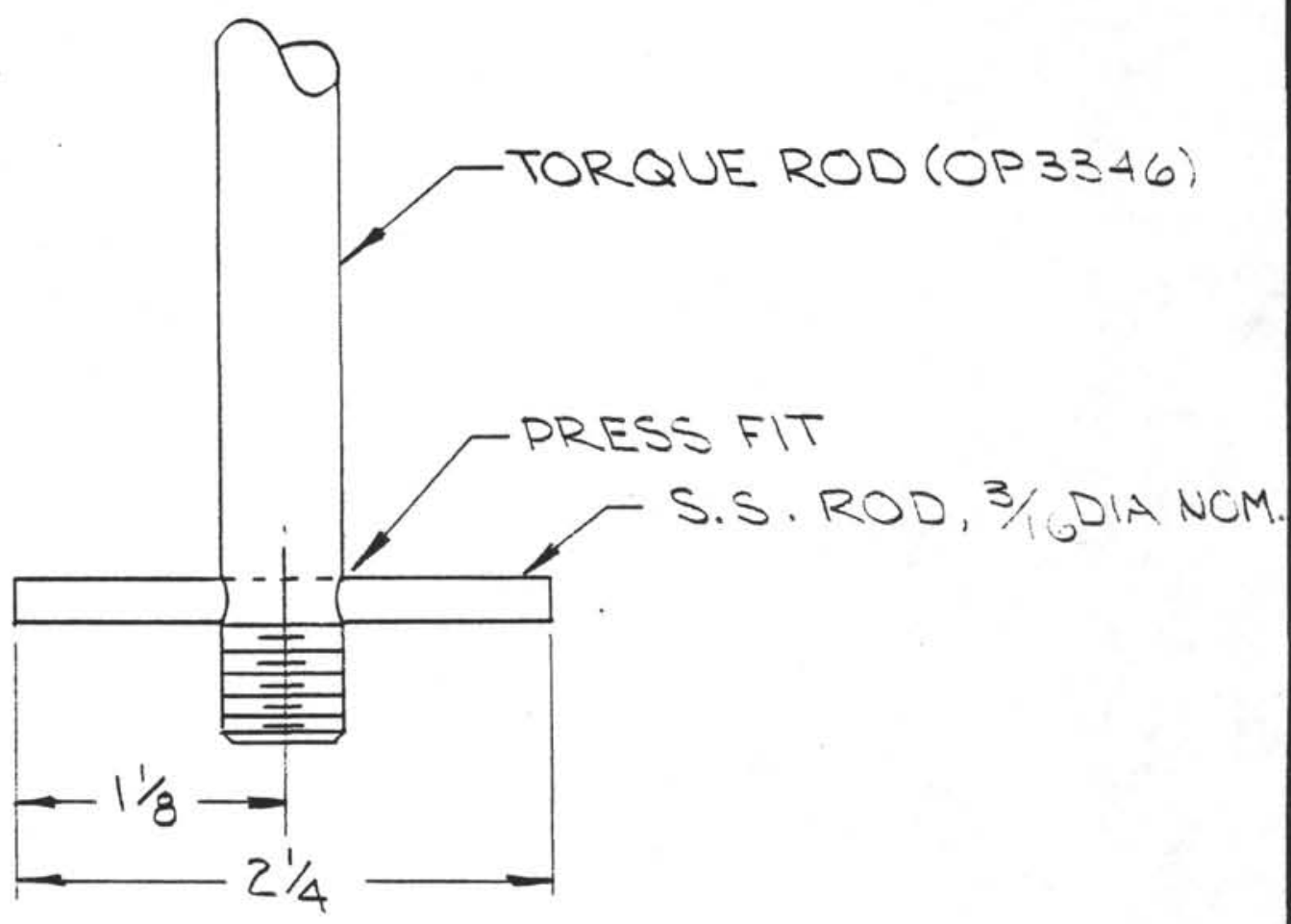

DO NOT SCALE TOLERANCES UNLESS NOTED

FRACTIONS $\pm 1 / 64$ DECIMALS \pm .005

ANGLES $\pm 1 / 2^{\circ}$ CORNERS $1 / 64 \times 45^{\circ}$ or $1 / 64 R$ FINISH 125 SURFACE TREATMENT $\frac{0}{\text { HEAT TREATMENT }}$ $\mathrm{O}$
CONCENTRICITY ALL DIAMETERS: TIR .003

\section{DEEP SEA DRILLING PROJECT}

SCRIPPS INSTITUTION OF OCEANOGRAPHY UNIVERSITY OF CALIFORNIA, SAN DIEGO

LA JOLLA, CALIFORNIA

TITLE

TORQUE ROD ASSY $\sim C B I P \sim$

MATERIAL

SEE ABOVE 4.18 .83

\begin{tabular}{l|l|l}
\hline SCALE & REQ'D/ASS'Y & PART NO.
\end{tabular}

OP 3357

DWG. NO

(REV)

$A-O P B=57$ 


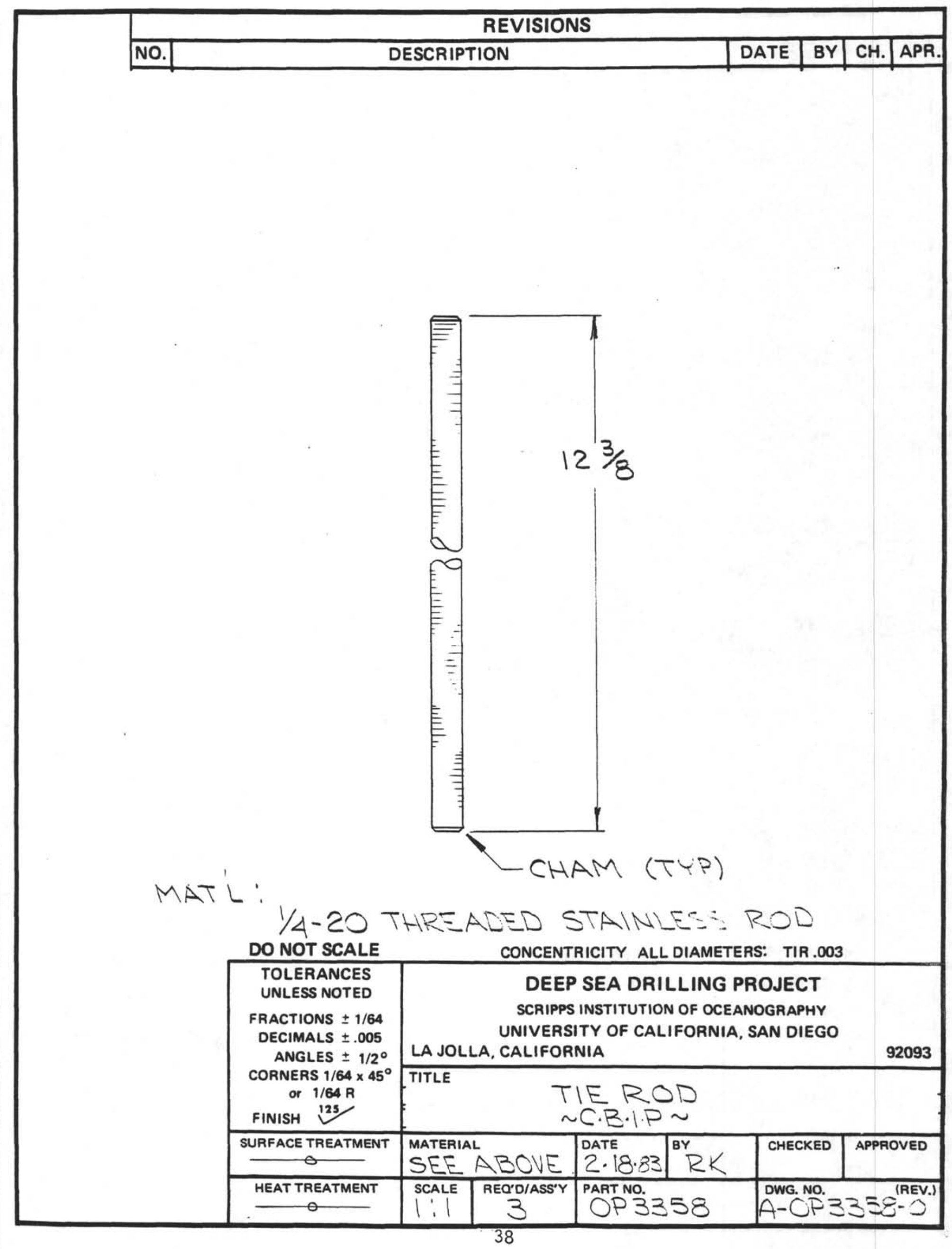




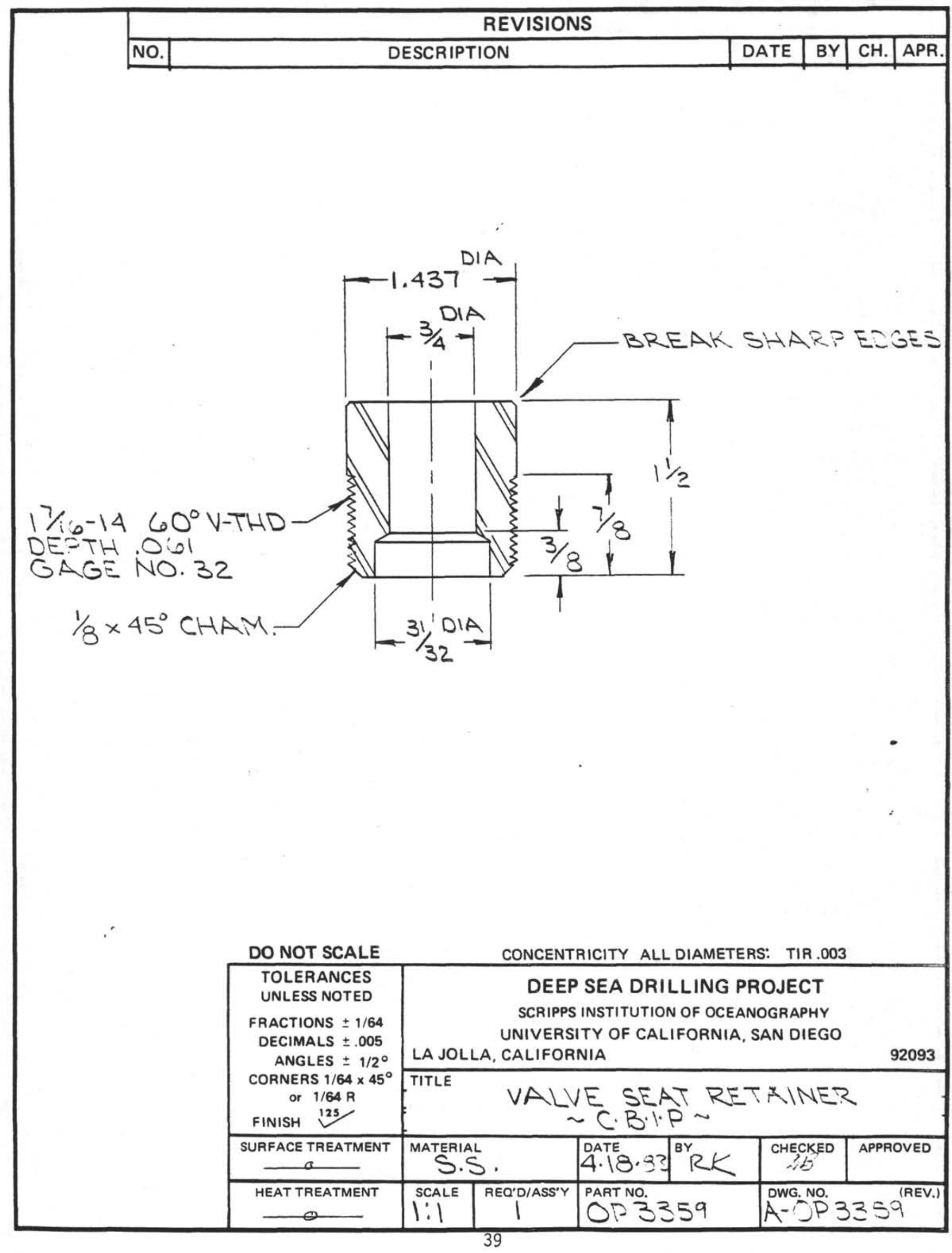



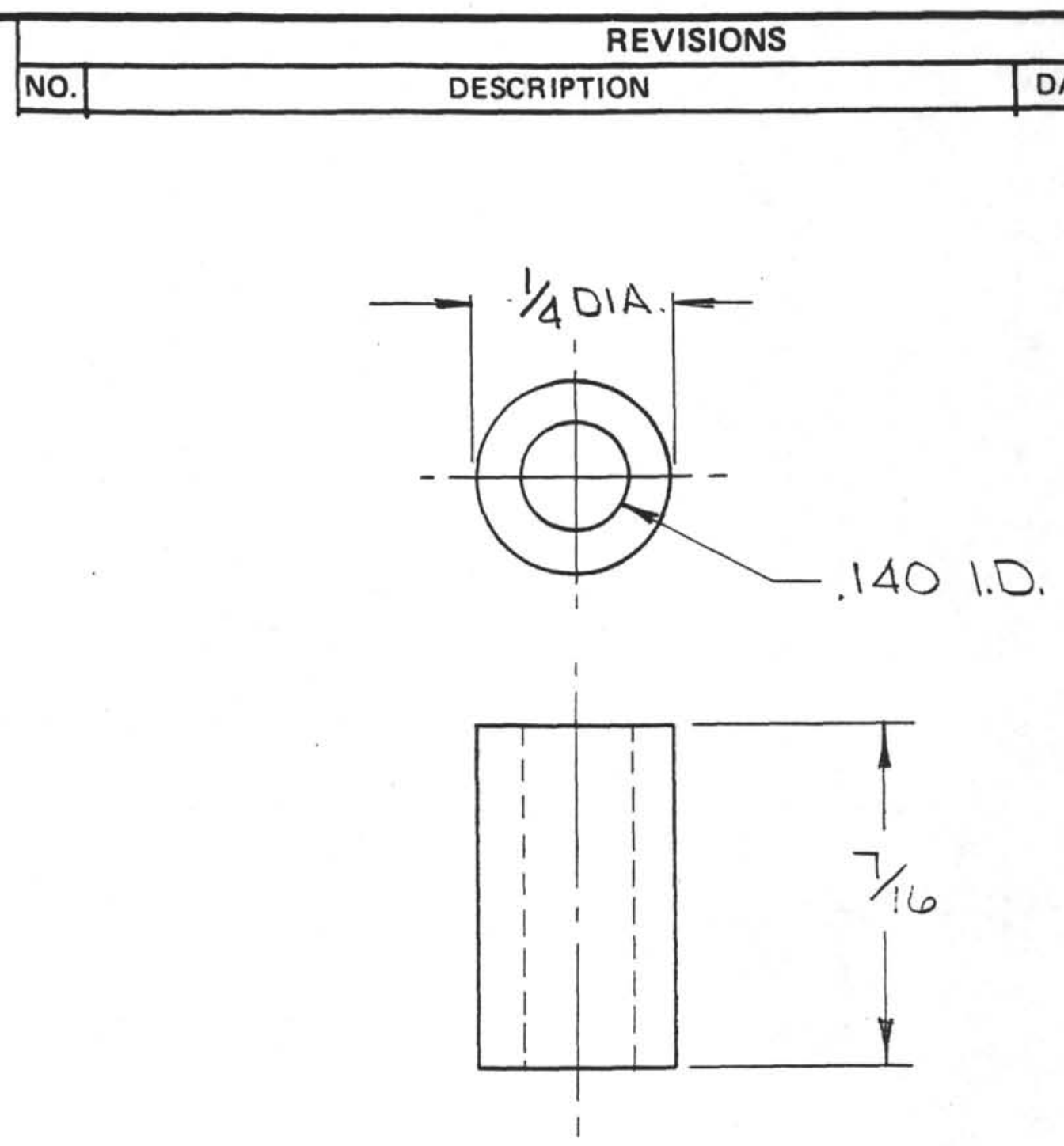

MAT:

META: OR PLASTIC

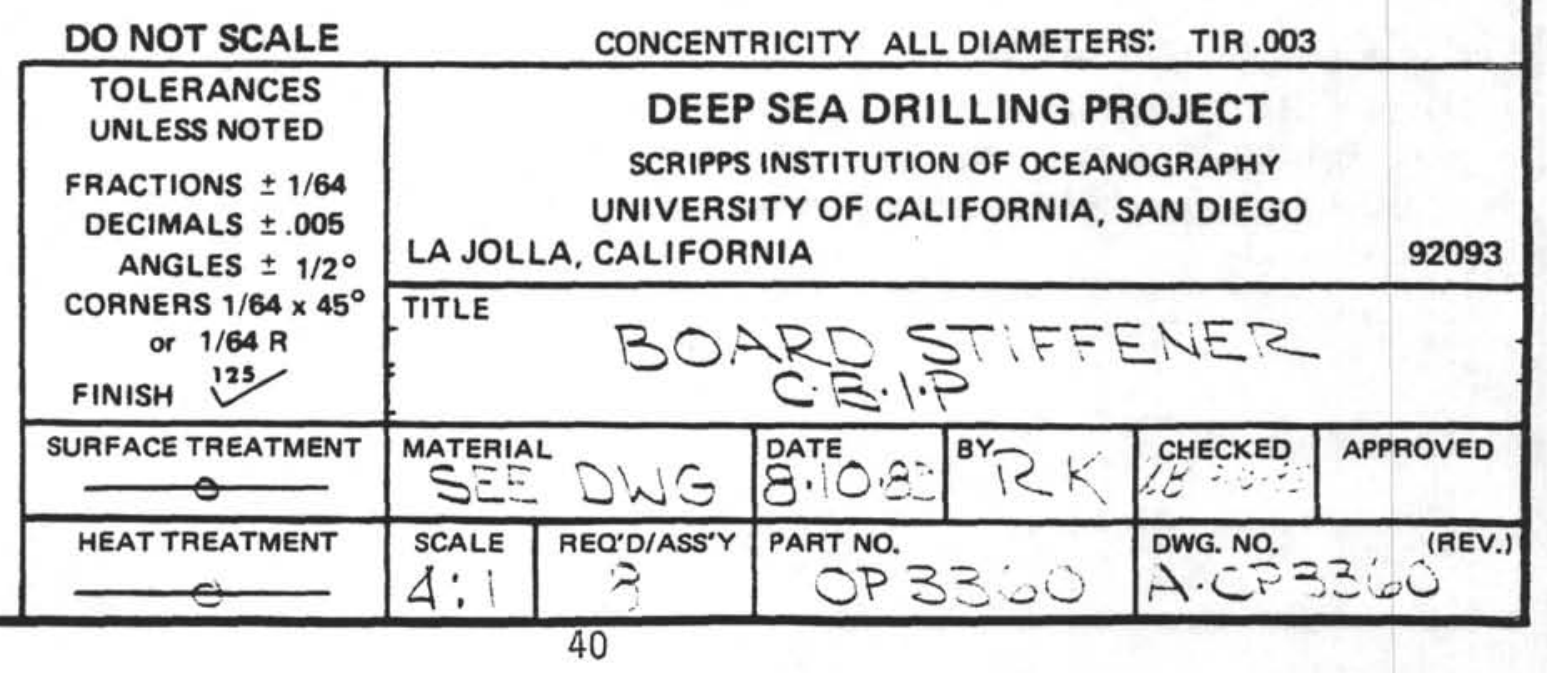




\section{APPENDIX A}

Pressure Measurement Tool Recorder Package Specifications 


\section{Fressure Measurement Tool}

Recorder Package

Specifications

Power Requirements

Memory

Sampie Rates

Data Output

Enviromental

Trarsducers
16 WDC, 5 Watts

16384 data points total. The number of samples is determined by the total number of inputs. (4 inputs = 4096 samples)

1 sample every $5.12,2.56$, $1.28, .64$, or $.32 \mathrm{sec}$. jumper selectable

12 bit binary (signed magnitude) TTL comp.

0 to $85 \mathrm{c}, 25 \mathrm{~g}$ shock, $10,000 \mathrm{psi}$

Be 11 and Howell CEC-1200 0 to 5 VDC outpui, 10,000 lbs max working pressure. 12 to 46 voc unregulated excitation, common to tool side of input plug.

2 to 6. Each transducer requires 1 wire. fi:l signsl low and exciter ground aits common to tool side ot piug 
Pressure Measurement Tool

Deck Interface

Specifications

Power requirements

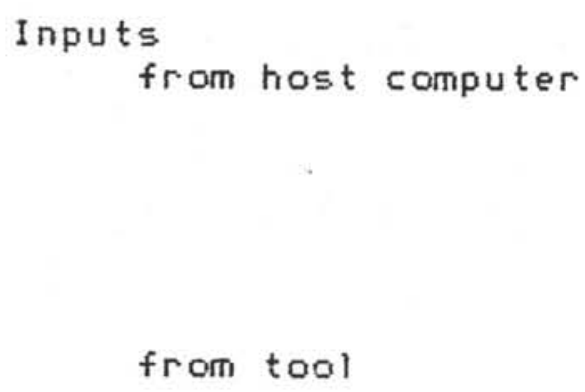

visual
120 VAC

.5 usec negative pulse to to initiate each byte of data transfer. Also data accepted. This is a pos. transition on completion of each byte transfekred.

12 bit byte of data, TTL or cMos compatible.

12 bit signed magnitude byte. Also Data Availabie (logic high when data is latched into interface).

Chip Enable -. This is a .5 uSec negative pulse from the host computer. Address step. This is the positive transition of data accepted.

Chif Enable -. 3 usec neg pulse.

Write Enable -. $20 \mathrm{~Hz}$

Start Convert. $20 \mathrm{~Hz}$ signal opposite Write enable. Address Step. Push button with channel select.

12 bit status indicators. (on $=+$ ) 
Pressure Measurement Tool

The recorder package is constructed of low power consumption C-Mos integrated circuits and a crystal controlled oscillator, for low battery drain and stability over long periods of time. The battery pack consists of eight 2 UDC lead acid cells hooked in series for a total of 16 VDC. Total deployment times in excess of 16 hours should be easily attained, with data retention times running into days. Sample rates are jumper selectable at 5.12 , 2.56, and .64 sec. per sample. These rates can.also be doubled by moving a jumper ta provide rates of $2.56,1.24$, and .32 sec. per sampie. The number of analog inputs is variable from two to six, and the logic must be configured to reflect the number in use by means of jumpers. Recorder delayed start is controlled by eight switches numbered 1 to 8 , corresponding to 10 to 70 minutes of delay, with number a being zero delay. The package has no power ONOFF stitch, so a power-ori reset circuit has been built in to insure the tool logic always starts in the proper state. In addition, a reset button is installed so that delayed start times can be accurately predicted, and readouts to the computer can be re-started. The analog to digital converters require a 0 to 5 VDC input voltage, however, provisions have been made for one or two amiplifiers to replace $A / D^{\prime}$ s for low level input signals. Since each low level input requires two $A / D$ slots, the total number of input channels will be reduced. The recorder package has 16384 
bytes of memory on-board, which must be divided by the total number of input channels to determine the number of samples taken. Four input channels will result in 4896 samples being recorded, six $=$ 2730 complete samples, with 2731 containing data from only the first four channels.

The deck interface unit is presently configured to output to a TEKTRONIX 4051 display terminal via the general purpose interface module plugged into the backplane. On receipt of a .5 usec negative pulse from the computer, one byte of data is transferred from the tool to the deck interface, then to the computer. This process is repeated until one complete sample has been loaded into the computer, then it is manipulated and displayed in engineering units. The cycle repeats until all data has been transferred from the tool, displayed, and hard copied. Data may be read from the tool as many times as desired without "wiping out" its memory. The deck interface is also used to calibrate the recorder package, and use of the computer is not necessary as the interface provides all lagic signals needed for caliuration. The recorder may be calibrated dynamically, with compressed gas or water, or statically with an external power supply. During calibration the selected input channel transducer voltage is converted to a digital byte and written to memory. It is then read out to the interface and displayed on the 12 bit status indicators. The reading can be converted to dynamic pressure or static voltage and the channal adjusted accordingly. Selection of the next channel is accomplished by pressing the STEF push button on the interface. 
The outside diameter of the electronics section is $2 "$, and length is not determined at this time, but should not excede $18^{n}$. This will allow use in many other applications. The electronics package, battery, pressure transducers, and shock absorber will be mounted on a split shell type chassis $3.0^{\prime \prime}$ in diameter and about 4" in length, to fit inside a $6^{\prime}$ innerbarrel pressure case. 
APPENDIX B

Transducer Specifications 


\section{ter 1200 spusered thin film High Output Pressure Tonsducep}

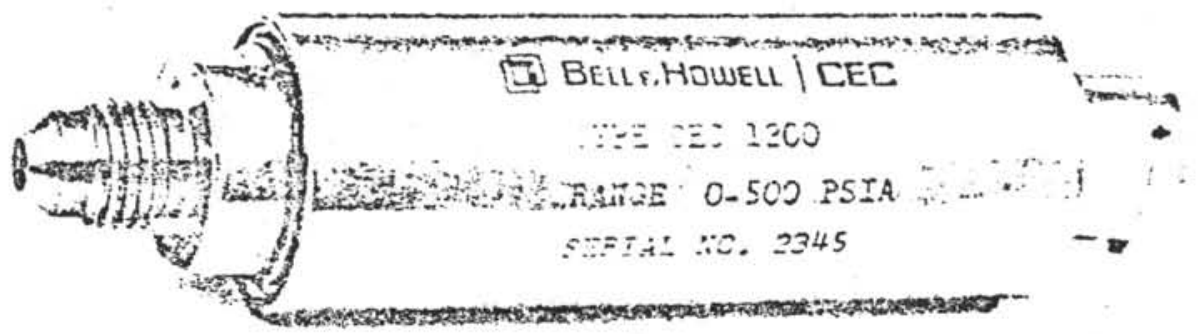

- Proven Sensor Design

- 5 Yolt Output

- Rugged Dual Cose isolotion

- Hioñ Performance

- Higfily Rellable

- Long-Term Stobility
Providing long-term stability and reliability, the CEC 1200 Sputtered Gage High Ovtput Pressure Transducer is a highly accurate thin iilm transducer. The thermal sensitivity error band performance is thoicaily better than $0.25 \%$ within any $50^{\circ} \mathrm{F}$ temperature band.

The use of spurtered film deposition and advanced design, combined with solid state signal conditioning for 5 volt output, create transducers with a maximum combined error for nonlinearity, hysteresis, and nonrepeatability of $=0.25 \%$ for the full range output.

Available in many standard ranges from 15 to $10,000 \mathrm{psi}$, the CEC 1200 also features an innovative double-case isolation. The basic sputtared sensor is electron beam weided to the pressure chamberiadapter, which also provides a high ciegree of mechanicai isolation from mounting torque eifects.

The CEC 1200, with integral amplifier, features a common negative input/output to provide three-wire operation. The unique amplifier design achieves a true zero output with reference to the common line.

The CEC 1200 Sputtered Gage High Output Pressure Transducers are manufactured in accordance with the program quality requirements of tillL-Q-9858A. 


\section{Pressure Roting}

Standard Ranges:

Burst Pressure:

\section{Electricol Characteristics}

\section{Excitation:}

Input Current:

Full Range Ouptut:

Residual Unbalance:

Output impedance:

Combined Nonlinearity,

Hysteresis, and

Nonrepeatability:

Insulation Resistance:

Electrical Connector:
$0-15,25,50,100,250,500,1000$. $1500,2000,2500,5000$ and 10,000 psi, absolute or gage. Sealed gage available in ranges of $100 \mathrm{psi}$ and above.

$200 \%$ of rated pressure, not to exceed 15,000 psi.

$300 \%$ of rated pressure, not to exceed 20,000 psi.

\section{Mechonicol Charocteristics}

Pressure Chamber Material: 17-4 PH stainless stee!.

Pressure Fiting:

Mounting Isolation:

\section{7:16-20 male, flared.}

Double case isolation provides assurance that the sensing eiement will be unaffected by external stress.

Sensing Element:

4 active-arm bridge.

Weight:

7 ounces maximum.

\section{Environmentol Performance}

\section{Temperature: \\ Operating Range: \\ Compensated Range: \\ Thermal Zero Shift: \\ Thermal Sensiuvity Shift: \\ Combined Thermal Zero and Sensitivity Shift: \\ Vibration Sensitivity:}

Natural Frequency:

Shock:

Humidity:

\section{Accessories}

Included:

Cptional: $-40^{\circ} \mathrm{F}$ to $+185^{\circ} \mathrm{F}$.

$-20^{\circ} \mathrm{F}$ to $+150^{\circ} \mathrm{F}$.

$=0.005 \%$ FRO $\% \mathrm{~F}$ nominal over the compensated temp. range.

$=0.005 \%$ FRO ${ }^{\circ} \mathrm{F}$ nominal over the compensated temp. range.

$0.3 \%$ over the compensated temperature range.

At $35 \mathrm{~g}$ peak from 10 to $2000 \mathrm{~Hz}$ (1/2* D.A. max.) the output shall not exceed $0.04 \%$ FRO $\mathrm{g}$ for 15 osi urits, decreasing logarithmicaliy to less than $0.002 \%$ - FRO g for 10,000 psi units. $50 \mathrm{kHz}$ at 5000 psi, decreasing logarithmicaliy to $5 \mathrm{kHz}$ at $15 \mathrm{psi}$.

Withstands $100 \mathrm{~g}, 11 \mathrm{msec}$ duration, hatt sine wave without damage.

Per MIL-E-5272C, Procedure 1.

Calibration record and dust caps. Mating eiectrical connector. (Specily CEC part number 166267-0005.)

\section{Ordering Information:}

When crdering, specily the instrument's complete type number. pressure range desired and whether absolute, gage, or sealed gage unit is required. (Example: CEC 1200 pressiste transducer, 0-100 psia.)

In keeping with Bell \& Howell's policy of continuing product improvement, specifications may be changed without notice. If the performance and configuration provided herein for our standard product dces not fit your exact needs, please check with us regarding customized transducers. Contact us directly at the factory or through your nearest CEC Sales Office.

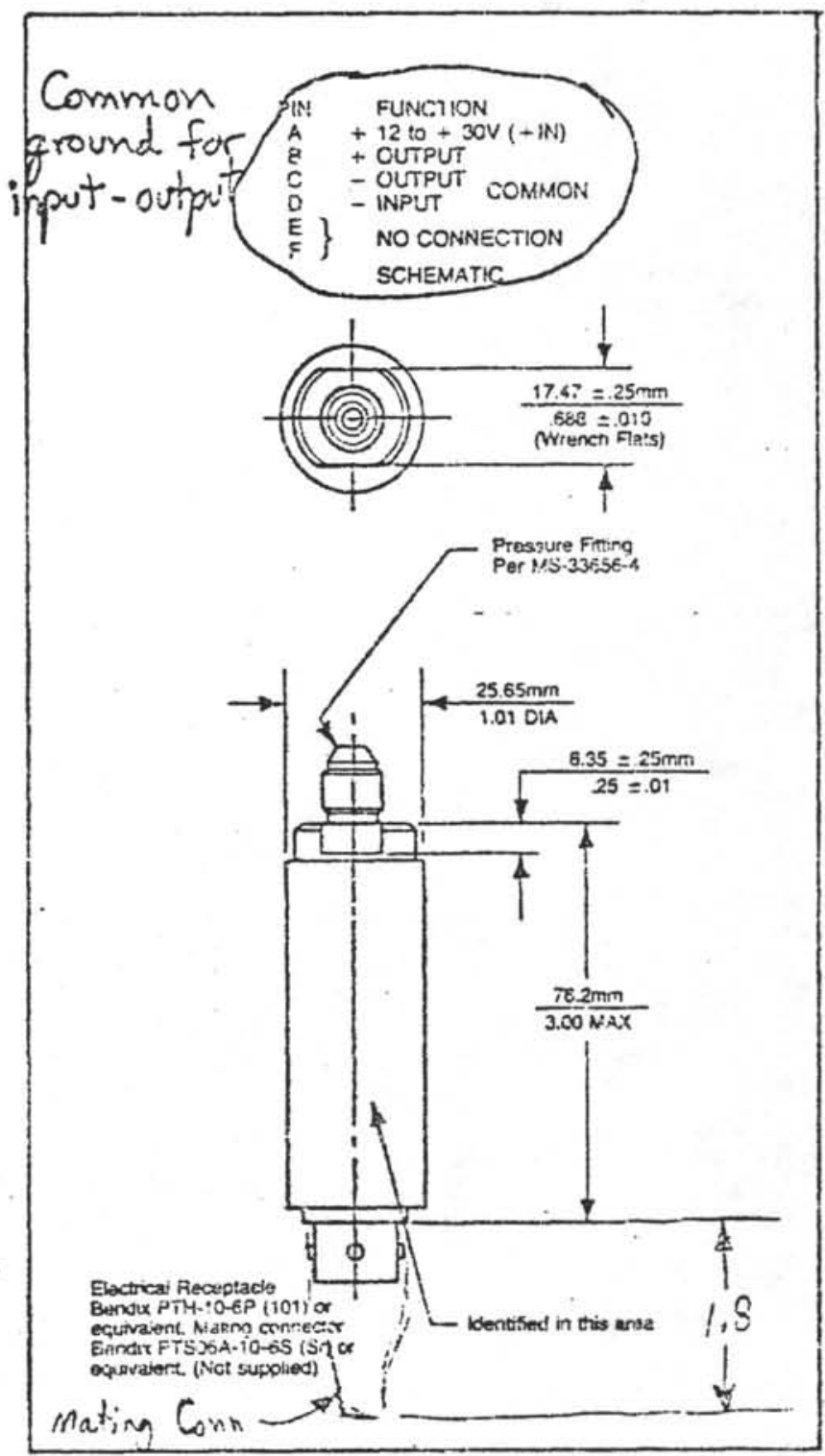

CEE DIVISIDN

360 Sierra Madre Villa, Pesadena, Calilornia 91109 (213) 796.9301 
APPENDIX C

Transducer Calibrations 


\section{CALIBRATION DATA}

\section{Instrument: CEC 1200}

Certified by:

\section{Equipment:}

$S / N \quad 1062$

Date: $4-28-83$

\begin{tabular}{|c|c|c|c|c|c|c|c|}
\hline \multirow{2}{*}{\multicolumn{2}{|c|}{$\frac{\text { S.N. } 1062}{P\left(\rho_{5}\right)} \vee V(0014)$}} & \multicolumn{2}{|c|}{ id 42} & \multicolumn{2}{|c|}{$\therefore 1050$} & \\
\hline & & $P$ & $V$ & $P$ & $V$ & & \\
\hline 0 & .0094 & 0 & .0043 & 0 & $=.0029$ & & \\
\hline 1000 & .517 & 1000 & .510 & 1000 & +.492 & $\therefore$ & \\
\hline 2000 & 1.022 & 2.000 & 1.014 & 2000 & .994 & & \\
\hline 3000 & 1.526 & 3000 & 1,516 & 3000 & 1.494 & & \\
\hline 4000 & 2. 230 & 4000 & 2.020 & 40000 & 1.293 & & \\
\hline 5000 & 2,530 & 5000 & 2.520 & 5000 & 2,490 & & \\
\hline 6000 & 3.030 & 60000 & 3.020. & 10000 & 2.290 & & \\
\hline 5000 & 2.5300 & 5000 & 2,5200 & 5000 & 2.420 & 0 & \\
\hline 4000 & 2.0300 & 4000 & $2,0 \geq 00$ & 4000 & 1.904 & .021 & \\
\hline 3000 & $1,526$. & 3000 & 1.520. & 3000 & 1.496 & .000 & \\
\hline 2000 & 1.0220 & 2000 & 1.020 .10 & 2000 & .926 & $0: 2$ & \\
\hline 1000 & .5170 & 1000 & .512 .6 & 1000 & .425 & .023 & \\
\hline $3 N$ id & 51 & 0 & .0059 .10 & 0 & -.0088 & .0311 & \\
\hline 0 & .0256 & & & & & & \\
\hline 1000 & .530 & & & & & & \\
\hline 2000 & 1.029 & & & & & & \\
\hline 3000 & 1.530 & $\therefore$ & & & & & \\
\hline 4000 & 2.030 & & & & & & \\
\hline 5000 & 2,530 & & & & & & \\
\hline 6000 & 3.030 & & & & & & \\
\hline 500 & 2.530 & 。 & & & & & \\
\hline 4000 & 2.030 & 0 & & & & & \\
\hline 3000 & 1.530 & 0 & & & & & \\
\hline 2000 & 1.0 .30 & .001 & & & & & \\
\hline 1000 & .530 & 0 & & & & & \\
\hline
\end{tabular}

\section{Full scale sensitivity:}

Shunt calib. data: 
APPENDIX D

MEASURED DATA 


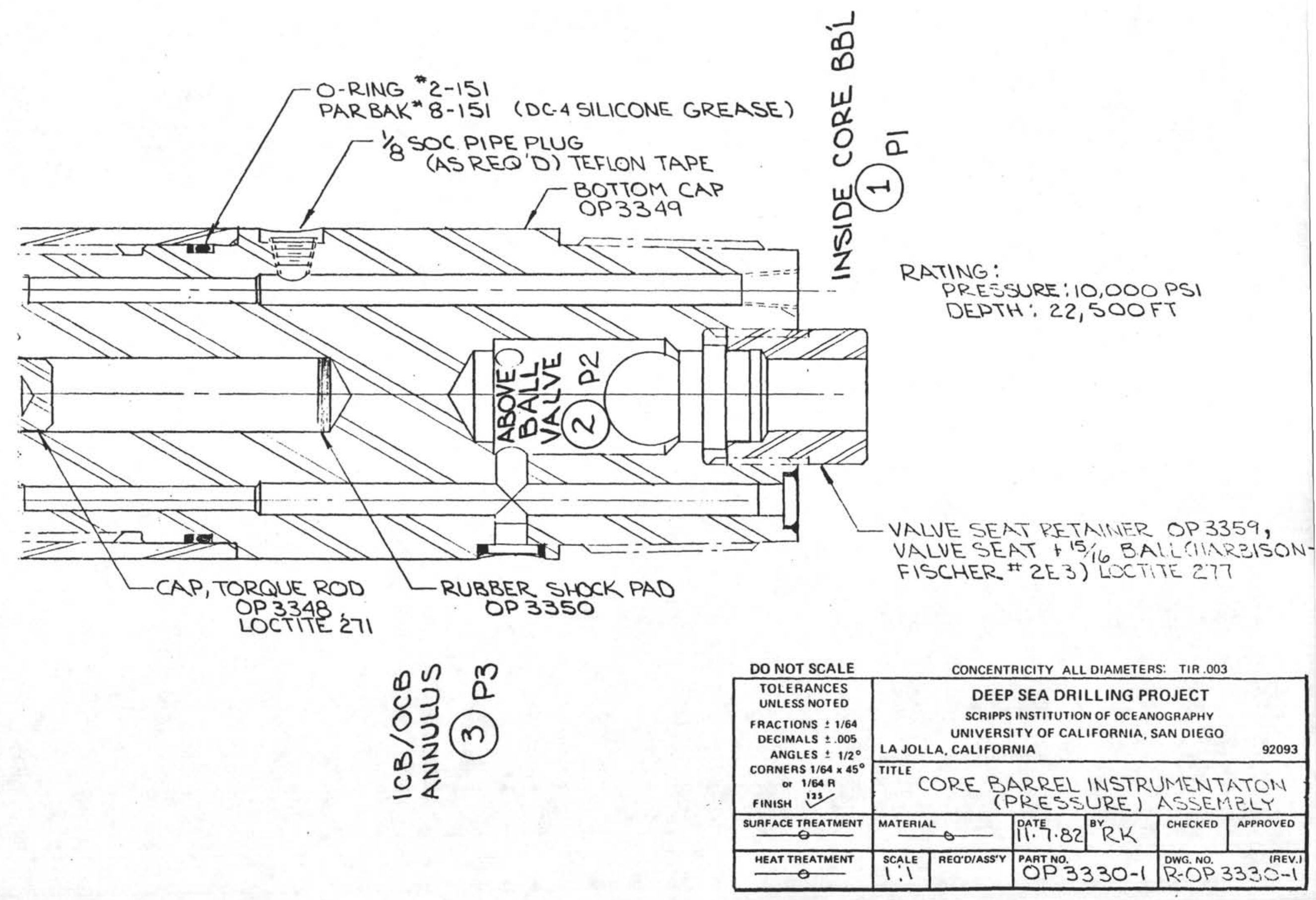


SIE 6/0 - WASH 3 BARRESS BETWEEN CORES $18+19$

D.C.

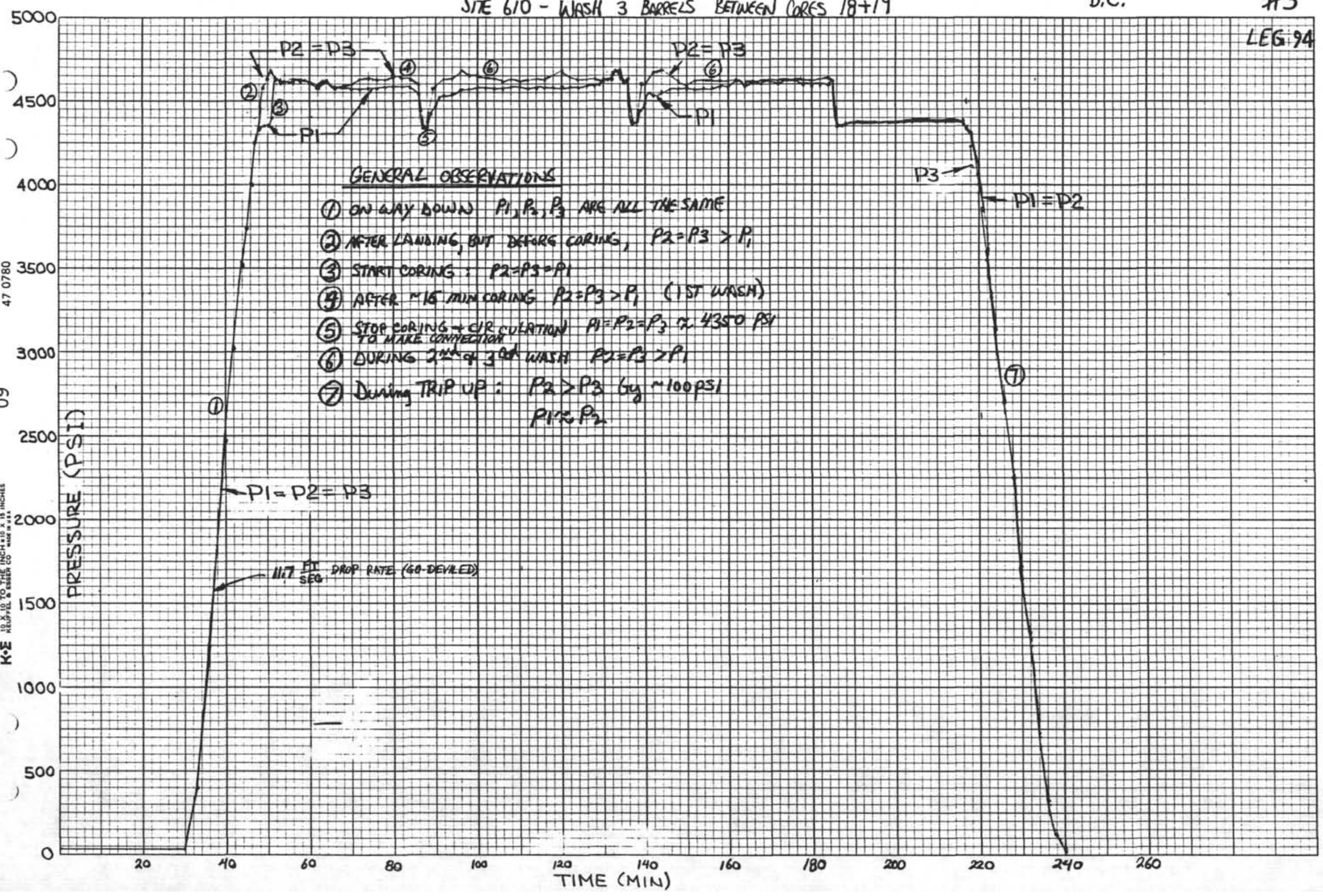



PRESSURE MEASURING TOOL

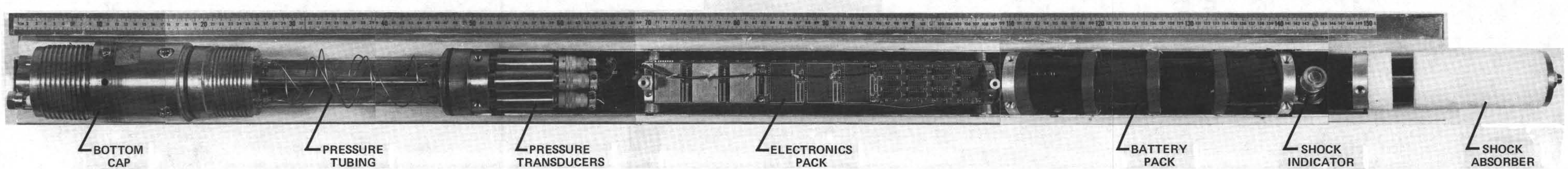

